\title{
On the Role of Suppression in Spatial Attention: Evidence from Negative BOLD in Human Subcortical and Cortical Structures
}

\author{
André D. Gouws, ${ }^{1}$ Ivan Alvarez, ${ }^{1}$ David M. Watson, ${ }^{1}$ Maiko Uesaki, ${ }^{1}$ Jessica Rodgers, ${ }^{1}$ and Antony B. Morland ${ }^{1,2}$ \\ ${ }^{1}$ York Neuroimaging Centre, Department of Psychology, University of York, York Y010 5DD, United Kingdom, and ${ }^{2}$ Centre for Neuroscience, Hull-York \\ Medical School, York Y010 5DD, United Kingdom
}

There is clear evidence that spatial attention increases neural responses to attended stimuli in extrastriate visual areas and, to a lesser degree, in earlier visual areas. Other evidence shows that neurons representing unattended locations can also be suppressed. However, the extent to which enhancement and suppression is observed, their stimulus dependence, and the stages of the visual system at which they are expressed remains poorly understood. Using fMRI we set out to characterize both the task and stimulus dependence of neural responses in the lateral geniculate nucleus (LGN), primary visual cortex (V1), and visual motion area (V5) in humans to determine where suppressive and facilitatory effects of spatial attention are expressed. Subjects viewed a lateralized drifting grating stimulus, presented at multiple stimulus contrasts, and performed one of three tasks designed to alter the spatial location of their attention. In retinotopic representations of the stimulus location, we observed increasing attention-dependent facilitation and decreasing dependence on stimulus contrast moving up the visual hierarchy from the LGN to V5. However, in the representations of unattended locations of the LGN and $\mathrm{V} 1$, we observed suppression, which was not significantly dependent on the attended stimulus contrast. These suppressive effects were also found in the pulvinar, which has been frequently associated with attention. We provide evidence, therefore, for a spatially selective suppressive mechanism that acts at a subcortical level.

Key words: contrast response; lateral geniculate nucleus; negative BOLD; spatial attention; suppression; visual cortex

\section{Introduction}

Spatial attention ensures that sensory processing is biased toward behaviorally relevant locations, allowing for enhanced perception of visual stimuli at attended locations. However, this benefit comes at the cost of impoverished perception of stimuli located elsewhere (Posner, 1980; Duncan, 2006). Potentially, attentiondependent enhanced perception could be underpinned by neural facilitation of signals at representations of attended stimuli while impoverished perception may result from suppression of neural activity at representations of unattended locations. However, the extent to which each of these effects is observed and the stages of the visual system at which these effects can be measured is not well understood.

While attentional enhancement is small or absent when the firing rates of single neurons are assessed in early visual structures (Motter, 1993; Luck et al., 1997), fMRI and ERP measurements in human appear to show larger attentional modulation of signals in primary visual cortex (V1; Gandhi et al., 1999; Martínez et al.,

\footnotetext{
Received Jan. 13, 2014; revised June 4, 2014; accepted June 20, 2014.

Author contributions: A.D.G. and A.B.M. designed research; A.D.G., I.A., D.M.W., M.U., J.R., and A.B.M. performed

research; A.D.G., I.A., D.M.W., M.U., J.R., and A.B.M. analyzed data; A.D.G. and A.B.M. wrote the paper.

The authors declare no competing financial interests.

Correspondence should be addressed to André D. Gouws, York Neuroimaging Centre, Department of Psychology,

University of York, York Y010 5DD, UK. E-mail: andre.gouws@ynic.york.ac.uk.

DOI:10.1523/JNEUROSCI.0164-14.2014

Copyright $\odot 2014$ the authors $\quad 0270-6474 / 14 / 3410347-14 \$ 15.00 / 0$
}

1999; Somers et al., 1999) and the LGN (O’Connor et al., 2002). However, there is greater agreement across different methodologies that attention enhances signals in extrastriate visual areas, with a consensus that attention-dependent enhancement of neural signals increases further up the visual hierarchy (Treue and Martinez-Trujillo, 2007; Boynton, 2011).

Attention-related suppression has been reported in V1 and even the LGN in an autoradiographic study of macaque monkeys (Vanduffel et al., 2000). It is also clear that baseline firing of single neurons in V4 and V2, but not V1, is lower when attention is switched from inside to outside the nonstimulated cell's receptive field (Luck et al., 1997). Similarly, fMRI studies have revealed attention-dependent inhibition (Slotnick et al., 2003) and negative BOLD signals in V1 (and nearby extrastriate areas; Tootell et al., 1998a, 1998b; Smith et al., 2000; 2004; Shmuel et al., 2002). It appears, therefore, that there is an important attentional role for suppression in early visual structures.

Studies evaluating suppression through negative BOLD responses in the human visual system have focused on visual cortex, characterizing either the task or the stimulus dependence of suppression. This leaves three important gaps in our knowledge. First, because there is evidence that facilitatory effects of attention are expressed at some, but not all, stimulus contrasts, there is a need to evaluate how suppression relates to task as a function of the characteristics of the stimulus. Second, the role of suppression, as measured in macaque, has not yet been characterized in 
B

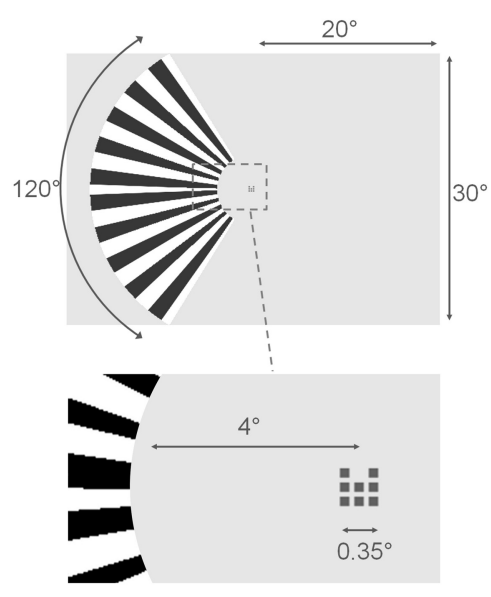

B

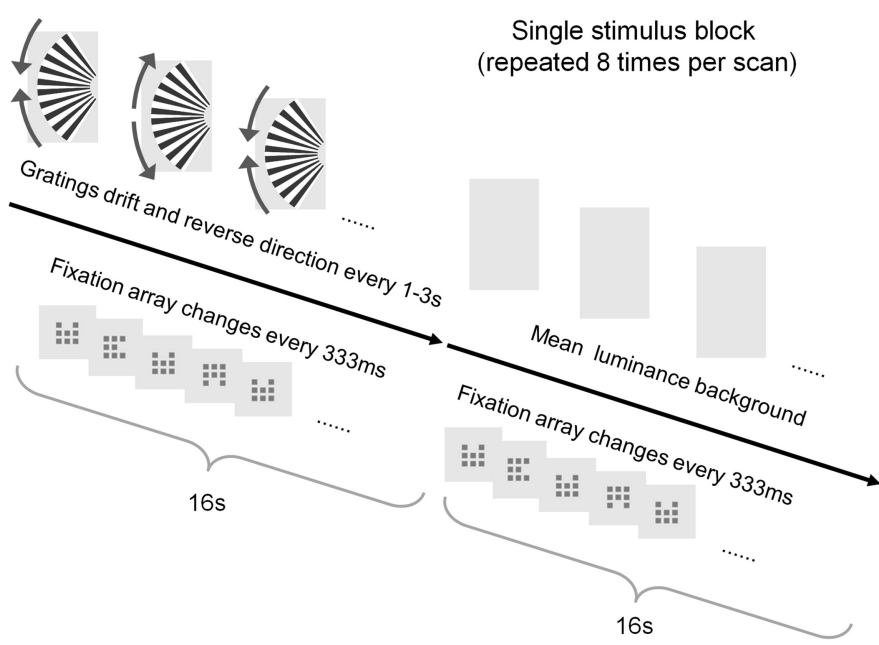

Figure 1. Stimulus configuration and timings. $\boldsymbol{A}$, The stimulus comprised two components in all experiments: a large, lateralized drifting grating and a dynamic fixation array of nine subsquares. $\boldsymbol{B}$, The central fixation array was presented throughout each scan, always changing every $333 \mathrm{~ms}$. The fixation elements were all red. The grating was presented for $16 \mathrm{~s}$, reversing direction randomly every 1-3s, and then removed to reveal the equiluminant background for $16 \mathrm{~s}$. Eight repeats of this $32 \mathrm{~s}$ cycle were included in each scan. Subjects either counted reversals of the grating, counted target changes at fixation, or viewed passively in separate sessions of four scans of the same task type.

human subcortical structures. Third, previous approaches to characterizing the LGN's contribution to attentional modulation have not been optimized to allow the contributions of suppression and enhancement to be disambiguated. This study aimed to characterize the dependence of negative BOLD signals on attention and stimulus contrast, asking the following questions: Is the attention-dependent neural suppression observed in macaque LGN also found in the LGN of humans? How does suppression in the thalamus and cortex depend on the stimulus characteristics of the attended stimulus and the task undertaken? Is the suppression expressed in the LGN and visual cortex also expressed elsewhere?

\section{Materials and Methods}

Two separate experiments were devised to characterize the task and stimulus dependence of visual responses in cortical and subcortical structures. The initial experiment was designed to assess solely the effect of task with high-contrast stimuli. A follow-up experiment was designed to examine the effects of task over a range of stimulus contrasts.

\section{Subjects}

Fifteen healthy subjects (nine female; mean age, 24.3 years) with normal or corrected-to-normal vision participated in the study after giving informed consent in accordance with the Declaration of Helsinki. The study was approved by the York Neuroimaging Centre Research Governance Committee (Department of Psychology, University of York). Ten subjects took part in the investigation of task dependence. Three of the initial 10 subjects and a further five new subjects took part in the subsequent investigation of contrast and task dependence of BOLD signals.

\section{Visual stimuli}

For the initial experiment (designed to assess solely the effect of task), high-contrast visual stimuli were rear-projected (Dukane Image Pro 8942 LCD projector) onto an acrylic screen in the bore of the MRI scanner. Lying supine, subjects viewed the stimuli via a front-silvered mirror placed above the head coil. The presentation of a lateralized visual stimulus can give rise to negative BOLD responses in the visual cortex ipsilateral to the stimulus. Given the size of the LGN, negative responses ipsilateral to a lateralized stimulus are more likely to be detected than those that might flank the positive responses in the LGN contralateral to the stimulus. With this in mind, we presented a large, high-contrast, lateralized, moving pattern to subjects to measure in the hemisphere contralateral to the stimulus positive BOLD responses that we could disambiguate from negative BOLD responses in the hemisphere ipsilateral to the stimulus. The stimuli were presented in the configuration summarized in Figure 1. Subjects maintained central fixation on a fixation array comprising $9 \times 9$ pixels $\left(0.35^{\circ}\right.$ visual angle $)$. The fixation array was divided into nine subsquares of $3 \times 3$ pixels each. The subsquare at the center of the fixation array was always on. Every $333 \mathrm{~ms}$, one of the eight surrounding subsquares would randomly disappear. The same surround subsquare could not disappear in consecutive intervals. The periodic change at fixation was used throughout the entire scan period of all fMRI datasets acquired. While fixating the central stimulus, the subjects were also presented with a peripheral stimulus comprising a $120^{\circ}$ sector of an annulus (inner radius, $4^{\circ}$; outer radius, $18^{\circ}$ ), equally divided by the horizontal meridian. Upper and lower halves of the peripheral stimulus were filled with drifting square-wave gratings (spatial frequency, 0.08 cycles per degree of polar angle; contrast, $100 \%$; frequency, $6 \mathrm{~Hz}$ ) that always had opposite motion directions (clockwise or counter-clockwise) and reversed unpredictably during a $16 \mathrm{~s}$ interval (block). Each $16 \mathrm{~s}$ block of peripheral stimulation was followed by a $16 \mathrm{~s}$ block where the fixation array alone was presented on the background (spatially uniform gray with luminance equal to the mean luminance of the gratings, $42 \mathrm{cdm}^{-2}$ ). Each of the 10 subjects underwent three experimental data-acquisition sessions; subjects were instructed to (1) fixate the central red stimulus throughout the scan (passive viewing task), (2) count the cumulative total of motion reversals of the peripheral stimulus (stimulus-related task), and (3) count the number of disappearances of the "12 o'clock" element in the fixation array (central task). For stimulus-related and central task conditions, subjects reported the count verbally after each scan. In each of the three scan sessions, four fMRI runs were acquired.

In the follow-up experiments to characterize the effect of stimulus contrast and task on BOLD responses, all software and hardware remained unchanged. Only two methodological changes were made from the initial experiment. First, the stimulus was respecified: the peripheral grating stimulus was modulated sinusoidally using only the red and green guns. The blue gun was set to zero because the blue value of some pixels in the projector array did not reproduce the selected stimulus level, giving rise to blue or yellow high- or low-lights, which could interfere with our measurements. The second methodological change from the initial experiment was a reduction in the number of tasks undertaken by each subject to just two: in each of eight sessions, eight subjects were required to complete either the previously described stimulus-related or central task, while the peripheral stimuli were presented at one of four contrast levels $(6,12,25$, or $100 \%)$. The passive task condition was thus omitted. Four repeat scans for each stimulus/task combination were ac- 
A

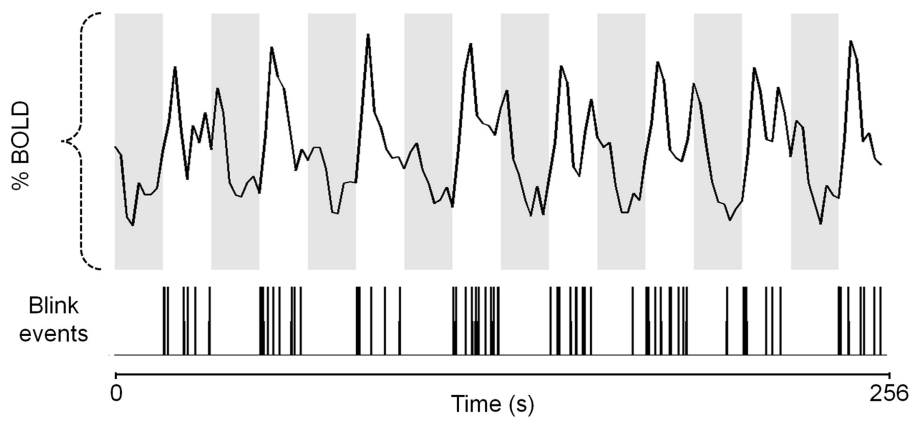

B

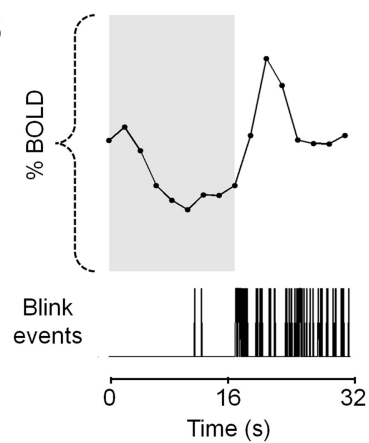

Figure 2. Negative BOLD responses may be correlated with systematic variations in blink activity. Mean data of four scans acquired from one participant under stimulus-related task conditions at $100 \%$ stimulus contrast. $A$, The mean $256 \mathrm{~s}$ BOLD time series from V1 ipsilateral to the stimulus. Gray and white backgrounds represent the periods of stimulus presentation and absence, respectively. Below the BOLD time series, we plot the blink events gathered from the corresponding eye video data. The majority of blink events appears to fall in the "grating-off" (white) period. $\boldsymbol{B}$, The data presented in $\boldsymbol{A}$ were averaged over the eight stimulus cycles to produce a mean BOLD response over a single-stimulus cycle with the corresponding blink distribution. Also apparent is a high density of blink events immediately following the offset of the grating stimulus (gray/white border), which is in turn followed by the pronounced overshoot of the BOLD response plotted above it.

quired in a single session. Condition order was counterbalanced across subjects. Across all measurements made, a single subject was always presented with stimuli in a single visual field. The visual field tested was counterbalanced across subjects.

\section{MRI acquisition}

Functional data. Data were acquired using a 3 tesla MRI scanner (Signa Excite, GE Healthcare) using an eight-channel phased-array head coil at the York Neuroimaging Centre. For fMRI acquisitions, gradient recalled echo pulse EPI sequences were used to measure T2* BOLD data (TR, 2000 ms; TE, 30 ms; FOV, $192 \mathrm{~mm}$; flip angle, $90^{\circ}$; $128 \times 128$ matrix; 22 contiguous slices with $2.4 \mathrm{~mm}$ slice thickness; voxel size, $1.5 \times 1.5 \times 2.4$ $\mathrm{mm}^{3}$ ). Para-axial slices were oriented to lie approximately parallel to the anterior-posterior commissure line, ensuring coverage of the occipital lobe. Magnetization was allowed to reach a steady state by discarding the first five volumes.

Structural data. We acquired proton density images (TR, $2520 \mathrm{~ms}$; TE, $35 \mathrm{~ms}$; flip angle, $90^{\circ}$; matrix size, $512 \times 512$; FOV, $192 \mathrm{~mm}$; 22 contiguous slices; slice thickness, $2.4 \mathrm{~mm}$; voxel size, $0.375 \times 0.375 \times 2.4 \mathrm{~mm}^{3}$; two averages) to allow the LGN to be identified (Devlin et al., 2006). Proton-density-weighted scans were acquired at the start of each session for each subject at the same slice orientation and prescription used to acquire the functional data. T1-weighted sagittal images (TR, $7.80 \mathrm{~ms}$; TE, $3 \mathrm{~ms}$; flip angle, $20^{\circ}$; matrix size, $256 \times 256$; FOV, $290 \mathrm{~mm}$; 176 slices; slice thickness, $1 \mathrm{~mm}$; voxel size, $1.13 \times 1.13 \times 1 \mathrm{~mm}^{3}$ ) were acquired to provide a high-resolution, whole-brain anatomical images and a canonical frame of reference to which all other functional and anatomical volumes were aligned.

\section{Defining regions of interest}

Proton-density-weighted anatomical images were assessed to provide volume definitions for the LGN regions of interest (ROIs) in all subjects using previously described criteria (Fujita et al., 2001; Devlin et al., 2006). Reassuringly, the resultant LGN tissue volumes estimated using this approach are highly comparable to the volumes reported in a histological assessment of human LGN volume (Andrews et al., 1997): the volume of our group mean left and right LGN ROIs were $128.2 \pm 12.64 \mathrm{~mm}^{3}$ and $138.0 \pm 13.59 \mathrm{~mm}^{3}$, respectively. V5/MT + can be reliably localized $1 \mathrm{~cm}$ superior to the junction of the inferior temporal sulcus (ITS) and the ascending limb of the ITS (AL-ITS; Dumoulin et al., 2000). Interpolated slices through a near-isotropic T1-weighted MRI volume $(1 \times 1.13 \times$ $1.13 \mathrm{~mm}^{3}$ ) were generated using custom software (Gouws et al., 2009), allowing bilateral identification of the junction between the ITS and the AL-ITS in all control subjects. The voxel $1 \mathrm{~cm}$ superior to this junction (along the AL-ITS) in the reformatted slice was used as the center of a spherical ROI of $1 \mathrm{~cm}$ radius. All subjects undertook fMRI retinotopic mapping procedures that allowed V1 to be identified. Specific stimulus and MRI acquisitions are specified here (Baseler et al., 2011). The T1- weighted structural scans for each subject were segmented into white and gray matter using mrGray (VistaSoft toolbox; http://white.stanford. edu/software/). The ROIs defined for V1 and V5 were transformed into this anatomical space within the VistaSoft toolbox. Only ROI voxels spatially corresponding to gray matter were retained for the final V1 and V5 ROI analysis.

\section{fMRI data analysis}

Under normal circumstances, fMRI analysis is largely restricted to using the stimulus as the explanatory variable, with the addition of other variables that seek to explain variance related to individual variations in hemodynamic responses. In the experiments we report here, the period during which the grating is off could elicit a greater number of blinks from subjects, particularly if this period follows a stimulus period in which the subject is performing a task. Blink-related signals have previously been discussed as potential sources of noise in fMRI measurements (Hupé et al., 2012), although the effects of systematic variation in blink pattern were not considered. Given these findings and our prediction that blinking may occur at systematically different rates during stimulus and control blocks, we sought to assess blinking in each subject. During all fMRI data acquisition, therefore, video data $(30 \mathrm{~Hz})$ were gathered for each subject's right eye via a long-range infrared camera (Eyetrack 6000 LR006, Applied Science Laboratories). Video files were post-processed offline. Initially, blinks were identified when the correlation of each video frame with its predecessor dropped below 0.8 . For quality control and verification of the algorithm's success, we subsequently manually assessed each video and found that the automated detection had an error rate of $2 \%$. Manually detected events were used to generate a timing file of each scan's blink events, which was later incorporated as an explanatory variable event file in general linear modeling (GLM) techniques to account for blinking patterns as a potential confound in our BOLD response estimates.

In Figure 2, we show an exemplar dataset supporting our concern that the timing of blink events is temporally correlated with the offset of an attended stimulus. It is clear in this example that the blink events are largely restricted to the period during which the grating stimulus is absent. It is also clear that the highest density of blink events is seen in the period immediately following the offset of the grating stimulus. Thus systematic variation in blink patterns, which have the ability to generate retinal and thus cortical signals, needs to be addressed as a potential confound in our estimates of BOLD responses, particularly in the context of negative BOLD. We therefore account for blinks in our fMRI analysis described below.

Functional data were corrected for within and across scan motion by aligning all volumes in the dataset to the first volume of the first scan of the session using MCFLIRT (a linear registration tool for motion correction of fMRI data; Jenkinson et al., 2002). We used the VistaSoft toolbox 
for Matlab (v7.8.1, MathWorks) to view unsmoothed data in a canonical anatomical framework. The time series had the linear trend removed (no other temporal filtering was applied) and was converted to percentage signal change. Mean time series for each scan were generated by first averaging the signal from all voxels within each of the ROIs and then averaging across the four scans that were acquired for each task/stimulus condition. We applied GLM to the time series data using the Matlab regression statistics function (regstats). Predictor time series were generated by convolving the SPM (http://www.fil.ion.ucl.ac.uk/spm/ software/spm8/) double-gamma hemodynamic response function (spm_hrf) with the stimulus and blink event timings and durations. Temporal derivatives of the predictors and a constant term were also entered into the GLM. The outcome measures used here are percentage signal change values for the stimulus and blink predictors. We ran independent GLMs for each contrast in the follow-up experiment when investigating responses at the stimulus representation. This allows for the effects of blinks to vary depending on the contrast presented, an appropriate precaution given the potential nonlinear interaction between stimulus and blink-driven activity. However, for ROIs without a representation of the peripheral stimulus, the time series at each contrast level were concatenated. We modeled, with a single GLM, the concatenated data with four stimulus predictors (one for each stimulus contrast) but only a single blink predictor (generated by temporally concatenating the blink predictors at each contrast level). This approach allows the stimulus predictor's contribution to vary across contrast levels as it should, but holds the blinks' contribution constant across all stimulus contrast levels. This approach is required because the effect of blinks should not be permitted to vary across conditions in regions that do not represent the stimulus.

Bootstrapping was used to estimate the mean contrast response function for each ROI and task condition. Each subject contributed a mean response value at each contrast level $(6,12,25$, and 100\%). Eight subjects were selected at random from the possible eight samples, with replacement. An estimate of the mean response at each contrast level was generated by averaging across these eight samples. A power function $R_{c}=$ $b C^{\gamma}$ was used to fit the four estimated means. $R$ is the measured response at a given input luminance contrast $C, b$ is a multiplicative constant, and $\gamma$ is an exponent. The fitting process was applied to multiple resampled datasets to generate $5 \times 10^{5}$ power functions, which allowed the modeled response maximum $R_{100}$ and semisaturation contrast $C_{50}$ to be derived. The frequency distributions of differences in these outcome measures resulting from different tasks were also computed and allowed the probability of a genuine difference to be estimated, as reported by the $p$ values in the manuscript.

Whole-brain GLM analyses were also conducted using FEAT (http://www.fmrib.ox.ac.uk/fsl). Motion correction was followed by spatial smoothing (Gaussian, FWHM $3 \mathrm{~mm}$ ) and temporal high-pass filtering $(0.01 \mathrm{~Hz})$. First-level analyses estimated the effects of stimulus and blink regressors (generated as before) for each scan. Additional steps included linear alignment of individual scan data into a canonical structural space, the MNI brain, using FLIRT (Jenkinson et al., 2002). Secondlevel, fixed-effects analyses generated estimates of within-individual mean responses. Third-level, mixed-effects analyses estimated the group mean responses. Statistical images were thresholded using clusters determined by $Z>1.63$ and a corrected cluster significance threshold of $p=$ 0.05 . To examine the thalamus, we masked the whole-brain group mean statistical images with a probabilistic thalamic atlas volume included with FSL4.1 (Behrens et al., 2003). This atlas divides the thalamus into seven distinct volumes defined by the probability of connectivity with various cortical regions. We were also keen to capture any activity in the superior colliculus so extended the thalamic ROI slightly to capture the top of the brainstem: a spherical volume of radius $8 \mathrm{~mm}$ was centered on the MNI coordinate $(1.0,-29.0,-2.0)$ and appended to the existing probabilistic thalamic mask. Only significant clusters falling within this volume are described in analyses.

\section{Behavioral data analysis}

We calculated the mean percentage error score for each participant as a function of the stimulus-contrast/task combination, for the stimulus- related and central task conditions (no counting task was performed under passive viewing conditions). For each individual scan, we first calculated the absolute difference between the reported number of target events and the presented target count, and converted this to percentage error value. This value was averaged across the four scans acquired for each individual subject for a given stimulus contrast/task combination. The resultant individual subject mean percentage errors were analyzed with a repeated-measures ANOVA to explore an effect of task alone in the initial experiment ( $n=10$, two task conditions at a single stimulus contrast). For the follow-up experiment, a repeated-measures ANOVA was applied to the individual subject mean percentage error values to explore main effects of task and stimulus contrast ( $n=8$, two tasks, four stimulus contrast levels).

\section{Results}

The first aim of our study was to determine whether negative BOLD responses could be detected in the LGN. While subjects attended to a large, lateralized drifting grating, i.e., under stimulus-related task conditions, there is clear evidence of negative BOLD responses in the time series derived from LGN ipsilateral to the stimulus (Fig. 3B, top). Moreover, and as expected, V1 ipsilateral to the stimulus also exhibited robust negative signals (Fig. 3C, top).

The stimulus-related task we used draws attention to the stimulus and attention has been shown to influence both positive and negative BOLD responses. To investigate the role of attention in modulating BOLD signals, we compared the results from the stimulus-related task data with our two additional conditions. Under passive viewing conditions, negative BOLD responses in the LGN and V1 ipsilateral to the stimulus appear reduced in amplitude relative to the stimulus-related task condition (Fig. $3 B, C$, middle), while having subjects perform a central task throughout the scan largely abolished the negative responses in those structures (Fig. $3 B, C$, bottom).

Positive BOLD responses were observed in the LGN, V1, and V5 contralateral to the stimulus (Fig. $3 D, E$ ), while V5 ipsilateral to the stimulus also exhibited positive BOLD responses (Fig. $3 E$ ), presumably because of the large receptive fields found in this cortical region (Zeki, 1969; Allman and Kaas, 1971; Amano et al., 2009). In the LGN and V1 contralateral to the stimulus, differences in task condition appeared to have little effect on positive BOLD responses. However, the magnitude of the positive BOLD responses found in V5 contralateral and ipsilateral to the stimulus was reduced for the passive viewing task and still further reduced for the central task compared with the stimulus-related task. It would appear therefore that early visual structures (the LGN and V1) contralateral to the stimulus exhibit positive BOLD responses that are modulated very little by attention, while their counterparts ipsilateral to the stimulus exhibit negative responses that are strongly affected by attention. The extrastriate motion area V5 exhibits positive BOLD responses that are strongly modulated by attention in both hemispheres.

The repeated-measures ANOVA revealed that subjects performed significantly $\left(F_{(1,9)}=58.32, p=3.20 \times 10^{-5}\right)$ worse on the central task (error rate, $7.78 \pm 1.01 \%$ ) than the stimulusrelated task (error rate, $0.99 \pm 0.37 \%$ ).

An interesting feature of the negative BOLD responses is a positive overshoot after stimulus cessation (Fig. $3 B$ ), especially noticeable under stimulus-related task conditions. Having seen the positive overshoot in pilot data, we hypothesized that it could be driven by an increase in blinking after stimulus cessation. We took the precaution therefore of video recording the subjects' eyes during our experiments to allow blinks to be detected (see Materials and Methods). An examination of how blink rate varies 

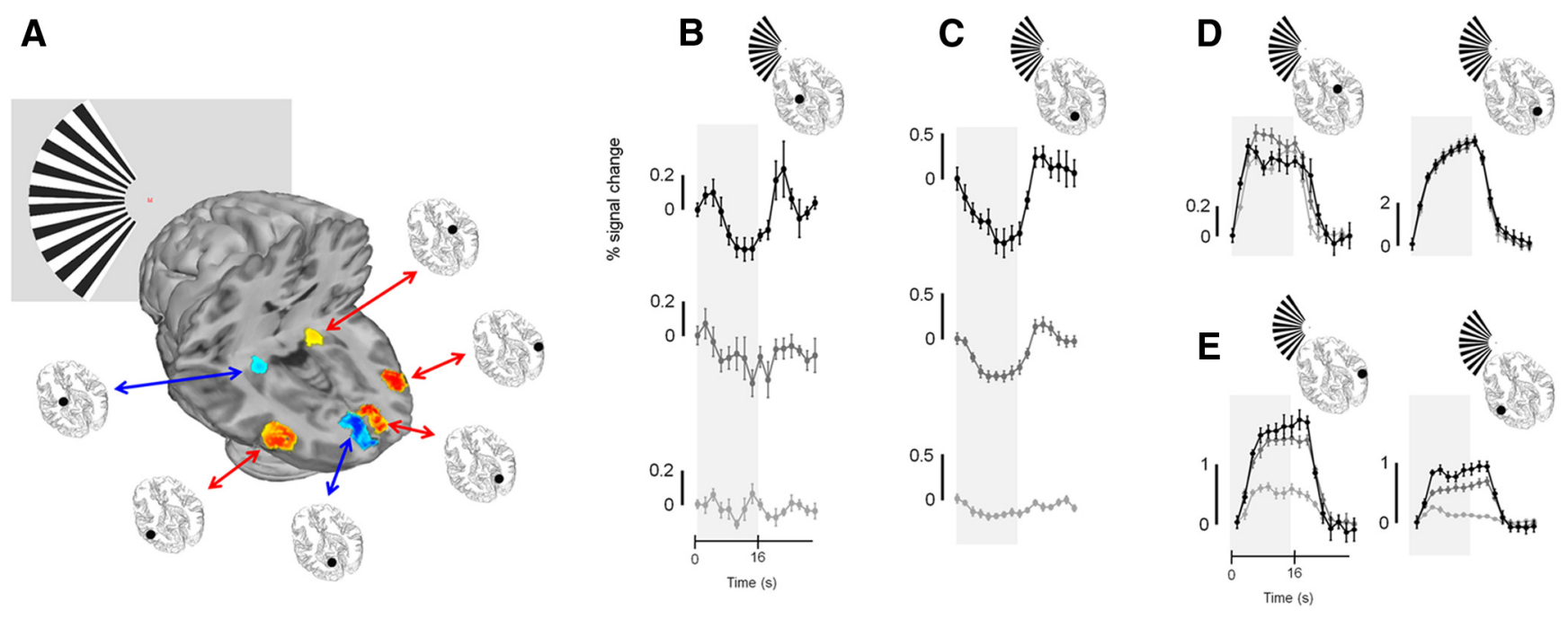

Figure 3. Group mean ( $n=10) \mathrm{fMRI}$ responses for the LGN, V1, and V5. $A$, Schematic of the stimulus configuration along with the locations of the LGN, V1, and V5 ROIs used to extract the time series data illustrated in $\boldsymbol{B}-\boldsymbol{E}$. $\boldsymbol{B}-\boldsymbol{E}$, Note that time series data are separated by condition in $\boldsymbol{B}$ and $\boldsymbol{C}$, but overlaid in $\boldsymbol{D}$ and $\boldsymbol{E}$. Time series data are given for the $L G N(\boldsymbol{B})$ and $V 1(\boldsymbol{C})$ ipsilateral to the stimulus for the stimulus-related (top, black), passive-fixation (middle, mid-gray), and central (bottom, light gray) tasks. $\boldsymbol{D}$, the responses obtained from the LGN (left) and V1 (right) contralateral to the stimulus. $\boldsymbol{E}$, the responses obtained from V5 contralateral (left) and ipsilateral (right) to the stimulus. The same shading of the lines and symbols in $\boldsymbol{B}$ and $\boldsymbol{C}$ are used in $\boldsymbol{D}$ and $\boldsymbol{E}$ to reflect the different task conditions. In all cases a single time series per subject was computed from four runs. The time series was then collapsed over a single stimulus cycle of $32 \mathrm{~s}$ for each subject. The single cycles were then averaged across all subjects to generate the responses plotted in the figure. Error bars indicate SEMs across subjects. The shaded gray region in all time series plots indicates the period during which the grating stimulus was presented.

during the stimulus cycle reveals that, after stimulus cessation, the group mean blink rate increases markedly for the stimulusrelated and passive viewing tasks, but not for the central task (Fig. $4 A$ ). It is vital therefore to account for any contribution that blink-related activity makes to the BOLD signals we measure. This can be done by performing GLM in which we use the stimulus as an explanatory variable (Fig. $4 B$ ) or both the stimulus and the blink rate as explanatory variables (Fig. 4C). For the group data, these two models were compared with hierarchical modeling. This analysis revealed that introducing blink rate as an explanatory variable accounts for additional variance, particularly in the LGN $\left(R^{2}\right.$ change, $0.23 ; F$ change, 28.762; $\left.p<10^{-10}\right)$ and V1 ( $R^{2}$ change, $0.044 ; F$ change, $12.835 ; p<10^{-5}$ ) ipsilateral to the stimulus. We also applied the same modeling approach to evaluate blink-related signals at the individual subject level because blink rates vary between subjects and tasks (Fig. 4A). This analysis revealed that in those subjects who increased their blink rate after stimulus cessation responses were partially, but significantly, driven by blinks. The quantitative analysis that we take forward for the rest of this study therefore accounts for the blinkrelated activity in all ROIs in each individual.

To examine the effect of task on cortical and subcortical visual signals, we derived the percentage BOLD response, specific to the stimulus, for each subject for all ROIs and task conditions (Fig. 5) using GLM techniques as described in the Materials and Methods section. We used one-way repeated-measures ANOVA to determine whether there were task-related changes in the BOLD responses we derived. We found significant main effects of task on the negative BOLD responses in the $\operatorname{LGN}\left(F_{(2,18)}=6.196, p=\right.$ $0.009)$ and $\mathrm{V} 1\left(F_{(2,18)}=15.32, p=0.00013\right)$ ipsilateral to the stimulus. The effect of task also followed a significant linear trend as a function of task (stimulus-related through passive viewing to central task $)$ in the $\operatorname{LGN}\left(F_{(1,9)}=17.68, p=0.002\right)$ and V1 $\left(F_{(1,9)}\right.$ $=18.91, p=0.002)$. Pairwise tests revealed significant differences between the stimulus-related and central tasks in the LGN ( $p=$ 0.007 ), and between the central task and both the stimulusrelated $(p=0.006)$ and passive viewing $(p=0.008)$ tasks in V1.
Task-dependent positive BOLD responses were also observed in V5 contralateral $\left(F_{(2,18)}=26.75, p=4 \times 10^{-6}\right)$ and ipsilateral $\left(F_{(2,18)}=18.68, p=4.1 \times 10^{-5}\right)$ to the stimulus. The responses decreased systematically with a linear trend as a function of task (stimulus-related through passive viewing to central task) in contralateral V5 $\left(F_{(1,9)}=68.21, p=1.7 \times 10^{-5}\right)$ and ipsilateral V5 $\left(F_{(1,9)}=22.67, p=0.001\right)$. Pairwise tests also revealed that the responses obtained from V5, contralateral and ipsilateral to the stimulus, under the central task differed significantly from the responses obtained under the other task conditions (contralateral V5: stimulus-related vs central, $p=5.1 \times 10^{-5}$; passive viewing vs central, $p=0.002$; ipsilateral V5: stimulus-related vs central, $p=0.003$; passive viewing vs central, $p=0.001$ ). In contrast, the positive BOLD responses in LGN and V1 exhibited no significant changes under the different task conditions $\left(\mathrm{LGN}: F_{(2,18)}=\right.$ $\left.2.169, p=0.143 ; \mathrm{V} 1: F_{(2,18)}=0.247, p=0.784\right)$.

The statistical analysis of the BOLD responses confirms the observations we made on the time series. In short, the LGN and V1 ipsilateral to the stimulus exhibit task-related negative BOLD responses, while we were unable to detect task-dependent changes in positive BOLD responses in the LGN and V1 contralateral to the stimulus. However, a strong task-dependent change in positive BOLD responses in V5 bilaterally was observed. Negative BOLD signals have been shown to be scaled inversions of positive BOLD responses (Shmuel et al., 2002). For this reason we decided to examine whether stimulus contrast, which strongly affects positive BOLD responses, would also affect negative BOLD responses in early visual structures. Also, taskrelated effects on positive responses in early visual structures have been reported to occur only at intermediate contrast levels rather than at the high contrast we presented (but see Gandhi et al., 1999; Li et al., 2008).

To determine the effect of contrast on the negative BOLD responses found in structures ipsilateral to the stimulus, we performed a follow-up experiment: we used a stimulus configuration identical to the one used in the initial experiment, but this time we presented sinusoidal grating components at different 
contrasts under only two of our previous task conditions, the stimulus-related and central tasks. Group mean time series for the LGN, V1, and V 5 contralateral and ipsilateral to the stimulus, at the four contrast levels tested $(6,12,25$, and $100 \%)$, are shown in Figure 6. Responses from V1 and the LGN ipsilateral to the stimulus exhibit markedly different responses compared with their counterparts contralateral to the stimulus (Fig. 6A,B). First, upward deflections are evident in the negative responses following stimulus cessation. As previously discussed, these reflect blink-related activity that is accounted for in our analyses. Second, the negative responses appear strongly dependent on task; only the stimulus-related task results in consistent negative responses, whereas the positive responses are observed under both task conditions. Third, negative responses appear largely independent of the stimulus contrast, showing that they are not simply scaled inversions of the positive, highly contrastdependent responses found in their contralateral counterparts (Fig. 6A, $B$, compare top left, top right). In V5, both contralateral and ipsilateral to the stimulus, positive responses are observed. As before, the stimulus-related task results in markedly larger responses than those obtained during the central task (Fig. 6C). The responses also appear to have only a weak relationship with stimulus contrast.

As before, we measured performance on both the stimulus-related and central tasks. The group mean error (1.04 \pm $0.21 \%)$ under stimulus-related task conditions was significantly $\left(F_{(1,7)}=23.89\right.$, $p=0.002)$ lower than during the central task $(5.78 \pm 0.90 \%)$. However, no significant effect of contrast $\left(F_{(3,21)}=1.405, p=0.269\right)$ or interaction between task and contrast $\left(F_{(3,21)}=2.002, p=0.145\right)$ was detected. Thus, for the follow-up experiment, participants again performed significantly worse on the central task than on the stimulus-related task, but performance did not change within task as a function of contrast.

To quantify the effects observed in the time series (Fig. 6) we estimated BOLD responses from each individual, for each condition, by applying GLM. As before, our model included each individual's blink pattern as an explanatory variable. The mean stimulus-related BOLD responses, for the group of eight subjects, are plotted as a function of contrast in Figure 7. We use repeatedmeasures ANOVA approaches with task and stimulus contrast as main effects to assess the data. In the LGN ipsilateral to the stimulus (Fig. $7 A)$, the effect of task $\left(F_{(1,7)}=6.170, p=0.042\right)$, but not contrast $\left(F_{(3,21)}=0.405, p=0.751\right)$, was significant. The interaction between task and contrast was not significant $\left(F_{(3,21)}=\right.$ 2.422, $p=0.094)$. A similar picture emerged for $\mathrm{V} 1$ ipsilateral to the stimulus (Fig. $7 B$ ); the negative responses were not significantly dependent on contrast $\left(F_{(3,21)}=0.990, p=0.417\right)$, but were dependent on task $\left(F_{(1,7)}=7.202, p=0.031\right)$ and there was no significant interaction $\left(F_{(3,21)}=1.679, p=0.202\right)$. Our find-
B
Stimulus-related

Passive viewing

Central
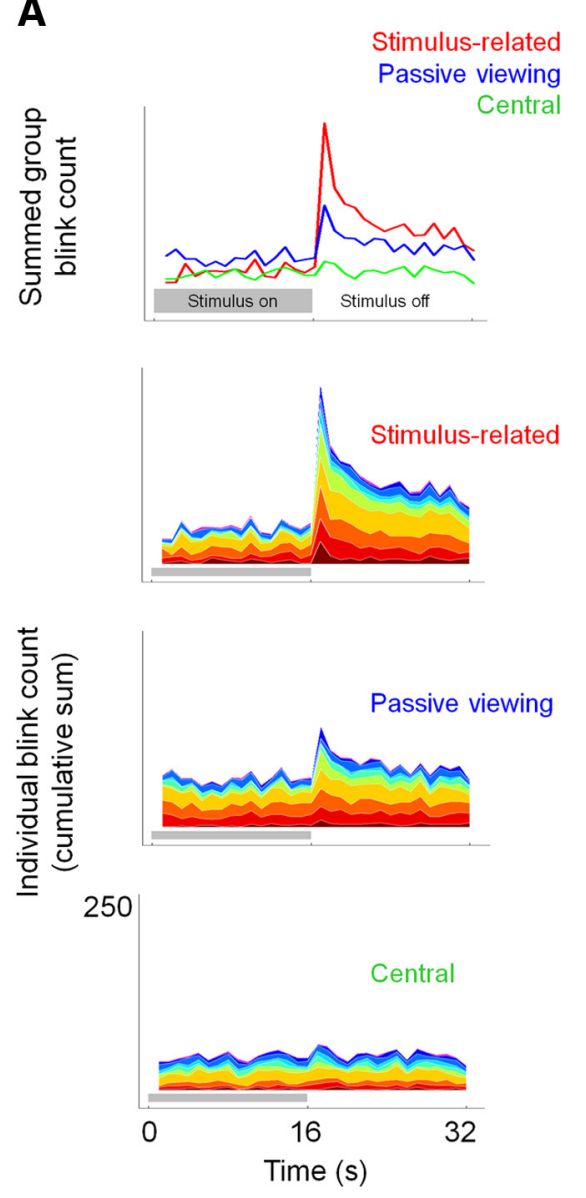

c

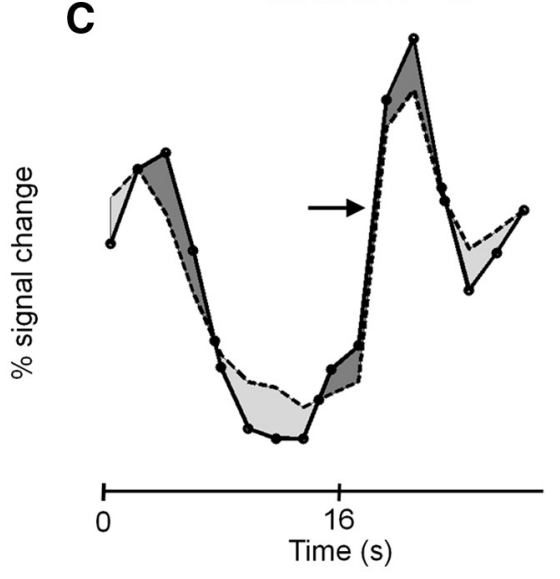

Figure 4. Blink rates and their effect on modeling BOLD responses. $A$, Top, How the number of blinks per TR $(2 s)$ varies over the time course of a single stimulus/control block of $32 \mathrm{~s}$. Data are summed across the subject group $(n=10)$ for three task conditions as indican the panel. The panels below (from top to bottom) show the contribution of each subject (different colors) to the variables only. Differences between the modeled and measured response (the residual error after modeling) are highlighted in gray. $\boldsymbol{C}$, The same data as shown in $\boldsymbol{B}$, but in this case the fit includes the blink rate as an explanatory variable. $R^{2}$ values are given for model fits (see text for details).

ing that negative BOLD signals in the LGN and V1 ipsilateral to the stimulus exhibit little or no contrast dependence requires careful comparison with previous results (Shmuel et al., 2002), which showed contrast-dependent negative BOLD signals in V1. Shmuel et al. evaluated the BOLD signals at the stimulus representation and in a region flanking it in V1. When we consider a similar region of $\mathrm{V} 1$ flanking the stimulus representation (Fig. $7 D)$, we too find negative BOLD signals that vary with contrast $\left[F_{(3,21)}=2.466, p=0.045\right.$ (one-tailed) $]$, but only when under the central task conditions, which match well with the task used by Shmuel et al. Interestingly, when a stimulus-related task is performed, we observed responses from this "flanking" region that are not significantly dependent on contrast $\left(F_{(3,21)}=0.284, p=\right.$ $0.837)$, mirroring the negative responses we found in $\mathrm{V} 1$ of the hemisphere ipsilateral to the stimulus (Fig. $7 B$ ).

In the LGN contralateral to the stimulus (Fig. $7 A$ ), the effect of contrast $\left(F_{(3,21)}=36.07, p=1.812 \times 10^{-8}\right)$, but not task $\left(F_{(1,7)}=\right.$ $0.014, p=0.909)$, was significant. The interaction was not significant $\left(F_{(3,21)}=0.054, p=0.983\right)$. In V1 contralateral to the stimulus (Fig. $7 B$ ), the highly contrast-dependent positive signals $\left(F_{(3,21)}=73.558, p=2.627 \times 10^{-11}\right)$ were dependent on task if, as justified on the basis of previous research showing only 

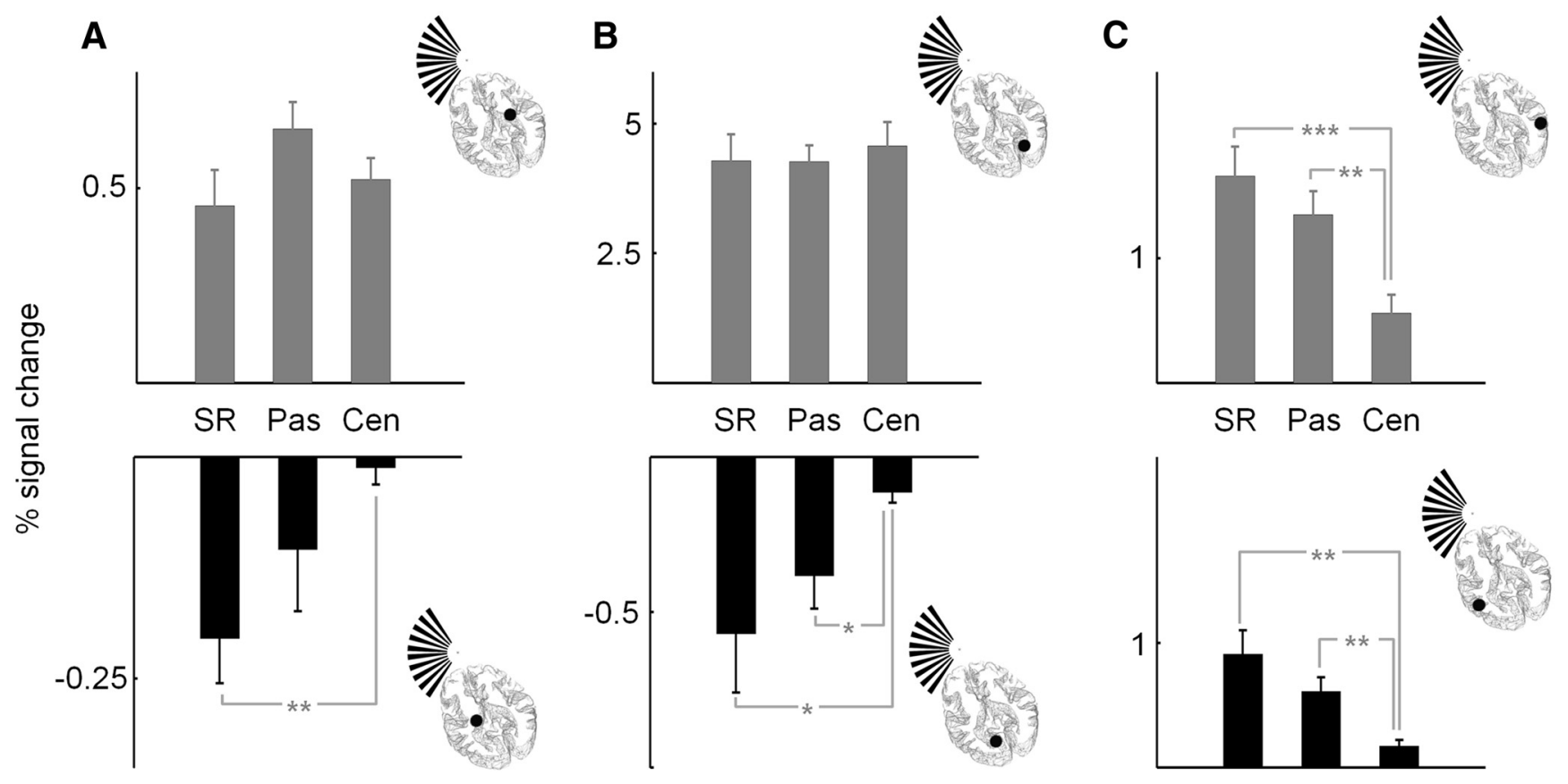

Figure 5. The effect of task on B0LD responses in the LGN, V1, and V5. $\boldsymbol{A}-\boldsymbol{C}$, The group mean stimulus-related activity is given for the LGN $(\boldsymbol{A}), \mathrm{V} 1(\boldsymbol{B})$, and V5 (C). In each panel, the data presented in the upper and lower plots illustrate the responses obtained from the brain regions contralateral and ipsilateral to the stimulus, respectively. Error bars are the SEM. Significant linear trends of response as a function of task were detected in all structures ipsilateral to the stimulus and in V5 contralateral to the stimulus (see text for details). Significant pairwise differences in responses are highlighted: ${ }^{* * *} p<0.001,{ }^{* *} p<0.01$, and ${ }^{*} p<0.05$ (two-tailed). SR, Stimulus-related task; Pas, passive viewing; Cen, central task.
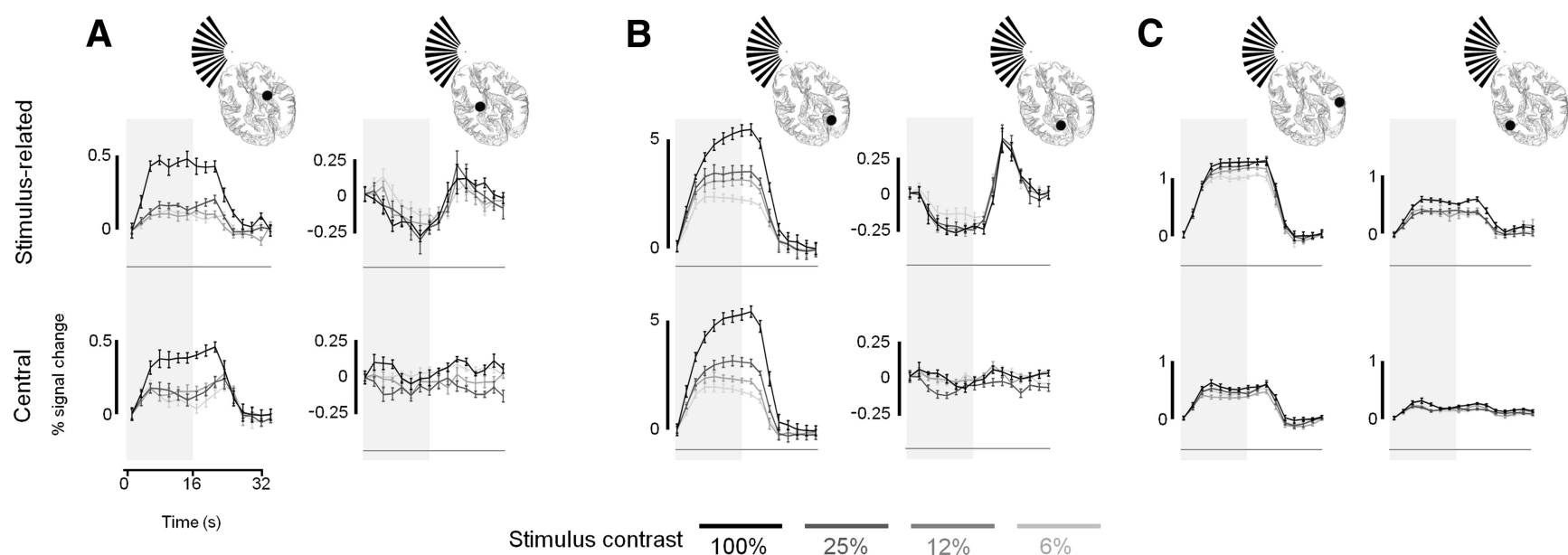

Figure 6. Group mean BOLD time series obtained from the LGN, V1, and V5 at different stimulus contrasts. $\boldsymbol{A}-\boldsymbol{C}$, Data are given for the LGN $(\boldsymbol{A})$, V1 (B), and V5 (C). In each panel the data for the hemisphere contralateral and ipsilateral to the stimulus are given in the left and right plots, respectively. Data are given for four contrast levels (as indicated by the key) and for stimulus-related task (top row) and central task (bottom row). Error bars indicate SEMs across subjects. Time series data are generated and presented as described in Figure 3.

attention-dependent increases in response, a one-tailed hypothesis is considered $\left(F_{(1,7)}=3.921, p=0.044\right)$. However, no significant interaction was detected $\left(F_{(3,21)}=1.557, p=0.229\right)$. In V5, both ipsilateral and contralateral to the stimulus (Fig. 7C), highly significant effects of task were observed (ipsilateral: $F_{(1,7)}=92.03$, $p=2.811 \times 10^{-5}$; contralateral: $F_{(1,7)}=224.8, p=1.409 \times$ $10^{-6}$ ) along with a subtle, but significant trend with contrast in the hemisphere contralateral to the stimulus (contralateral: $F_{(1,7)}$ $=9.316, p=0.019$; ipsilateral: $\left.F_{(1,7)}=1.506, p=0.259\right)$. For V5, no significant interaction of task and stimulus contrast was detected in either hemisphere (contralateral: $F_{(3,21)}=0.192, p=$ 0.901 ; ipsilateral: $\left.F_{(3,21)}=1.510, p=0.241\right)$.

There is the strong a priori evidence from fMRI for contrastdependent and task-dependent responses in the stimulus repre- sentations of the LGN (Kastner et al., 1999) and V1 (Buracas and Boynton, 2007). The use of repeated-measures ANOVAs is appropriate for evaluating unknown response relationships with contrast and task, like those we have assessed in the LGN and V1 ipsilateral to the stimulus. However, ANOVAs are not as sensitive as modeling techniques used previously to characterize the effects of task on the largely predictable contrast-response relationships obtained from early visual structures (Buracas and Boynton, 2007; Li et al., 2008; Murray, 2008). To increase our sensitivity to subtle task-dependent changes, particularly in the contrast responses obtained from the LGN and V1 contralateral to the stimulus, we extended our analysis to include the same modeling approach as others (Buracas and Boynton, 2007; Li et al., 2008; Murray, 2008). Specifically, we used a power function (Buracas 

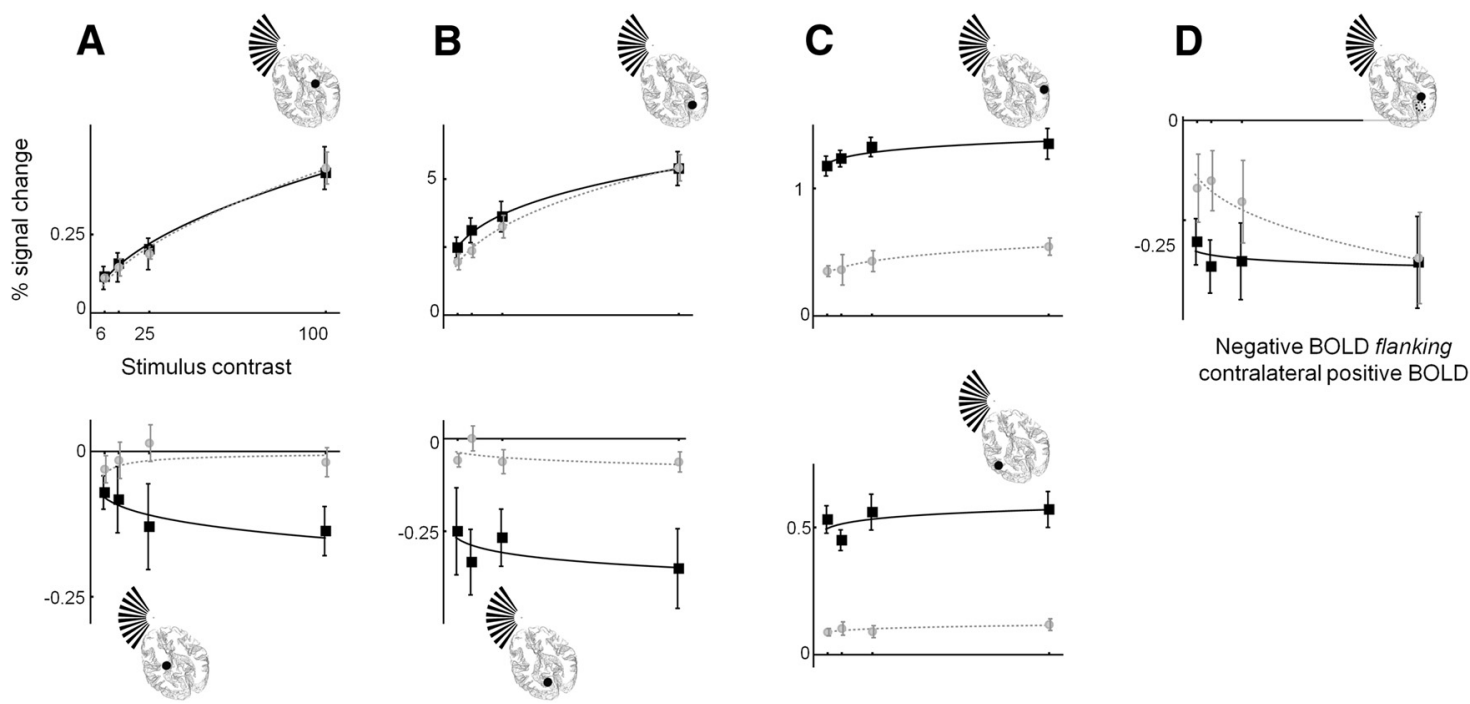
contralateral positive BOLD

Figure 7. Contrast responses for different visual structures obtained under stimulus-related (black squares, solid black lines) and central (gray circles, dashed gray lines) task conditions. $A-C$, Data are given for the LGN $(\boldsymbol{A}), \mathrm{V} 1(\boldsymbol{B})$, and V5 ( $\boldsymbol{C}$ contralateral (top row) and ipsilateral (bottom row) to the stimulus. $\boldsymbol{D}$, Data for the region of V1 in the hemisphere contralateral to the stimulus that flanks the stimulus representation. The lines through the data were generated by fitting a power function to the data as described in the text. Error bars indicate SEMs of the mean across subjects.

Table 1. $R_{100}$ and $C_{50}$ values for positive BOLD regions of interest: the LGN, V1, and V5 ${ }^{a}$

\begin{tabular}{|c|c|c|c|c|}
\hline \multirow[b]{2}{*}{ Outcome measure } & \multicolumn{3}{|l|}{ Contralateral } & \multirow{2}{*}{$\begin{array}{l}\text { Ipsilateral } \\
\text { V5 }\end{array}$} \\
\hline & LGN & V1 & V5 & \\
\hline \multicolumn{5}{|l|}{$C_{50}$} \\
\hline Stimulus-related & $29.1 \%(12.0-39.3 \%)$ & $7.99 \%(6.00-10.6 \%)^{b}$ & $0.01 \%(0.00-0.02 \%)$ & $0.01 \%(0.00-0.02 \%)$ \\
\hline Central & $24.8 \%(17.4-33.1 \%)$ & $16.10 \%(11.7-21.7 \%)^{b}$ & $1.20 \%(0.01-4.65 \%)$ & $0.19 \%(0.00-1.96 \%)$ \\
\hline \multicolumn{5}{|l|}{$R_{100}$} \\
\hline Stimulus-related & $0.37 \%(0.26-0.47 \%)$ & $4.58 \%(3.7-5.6 \%)$ & $1.31 \%(1.17-1.49 \%)^{b}$ & $0.64 \%(0.55-0.73 \%)^{b}$ \\
\hline Central & $0.39 \%(0.31-0.47 \%)$ & $4.76 \%(4.0-5.5 \%)$ & $0.56 \%(0.45-0.67 \%)^{b}$ & $0.11 \%(0.08-0.14 \%)^{b}$ \\
\hline
\end{tabular}

${ }^{a}$ Data are given for the stimulus-related and central tasks for structures contralateral to the stimulus for the LGN and V1, and bilaterally for V5. Mean modeled values are given with $95 \%$ confidence range in parentheses.

${ }^{b}$ Significant effects of task between conditions.

and Boynton, 2007) to fit the contrast response functions from the LGN and V1 contralateral to the stimulus (see Materials and Methods). This approach also allows for a more direct comparison of our results with those from earlier studies. From the resultant model fits, we derived two outcome measures: the modeled response at $100 \%$ contrast, $R_{100}$, and the contrast, $C_{50}$, at which the modeled response reaches half $R_{100}$. We used resampling techniques to establish whether these outcome measures were significantly affected by task (see Materials and Methods).

We summarize the results of power function fitting to all positive BOLD regions of interest in Table $1 . C_{50}$ and $R_{100}$ values are given for the LGN and V1 contralateral to the stimulus, and for V5 bilaterally. In the LGN contralateral to the stimulus, we detect no effect of task on either outcome measure. An effect of task on $C_{50}(p=0.008)$, but not $R_{100}$, was observed in V1 contralateral to the stimulus. In contrast, V5 exhibited a significant attentionrelated increase in $R_{100}\left(p<5 \times 10^{-4}\right)$, but not $C_{50}$. This pattern was also a feature of the attention-related effect in V 5 ipsilateral to the stimulus $\left(R_{100}: p<5 \times 10^{-4}\right)$. The quantitative comparisons reveal that the response change in $\mathrm{V} 1$ caused by attention shows increased responses at intermediate, but not maximum, stimulus contrast, a pattern commonly referred to as contrast gain. Attention-dependent changes of $\mathrm{V} 5$ responses on the other hand can be attributed to either increased baseline or response gaintype mechanisms.

Up to now we have shown that BOLD signals in the LGN and $\mathrm{V} 1$ are reduced compared with baseline in representations of unattended locations in the stimulus-related task. We believe the decrease in BOLD signal relates to a suppression of neural responses at representations of unattended locations in the LGN and V1. If this is the case, such suppression should also be present in representations of unattended locations when our central task is performed. But, because the central task is performed throughout stimulus and nonstimulus blocks, the neural firing will be constant across all blocks and will therefore not be detected with conventional fMRI analysis. However, we are able to take an alternative approach to examine whether suppression is present during the central task: immediately before acquiring our fMRI time series, we acquired dummy volumes that allow magnetization to reach a steady state. These volumes are automatically discarded by the scanner. During the dummy acquisitions, subjects fixated passively and only started to perform the central task following the dummy acquisitions. If neural suppression drives the decreases in BOLD signals we record, a negative deflection in the early part of the time series should be evident for the ROIs that represent unattended locations where no stimulus is presented. We found evidence for this initial negative deflection in the time series obtained from the LGN and V1 across all contrast conditions (Fig. $8 A, C$ ). To add context, we show how the initial negative deflection compared with the distribution of BOLD amplitudes recorded after the first stimulus cycle (Fig. 8B,D). A clear pattern emerges across contrast conditions. Specifically, the first and fifth temporal samples of the time series frequently represent the maximum and minimum recorded amplitude, respectively. 
A

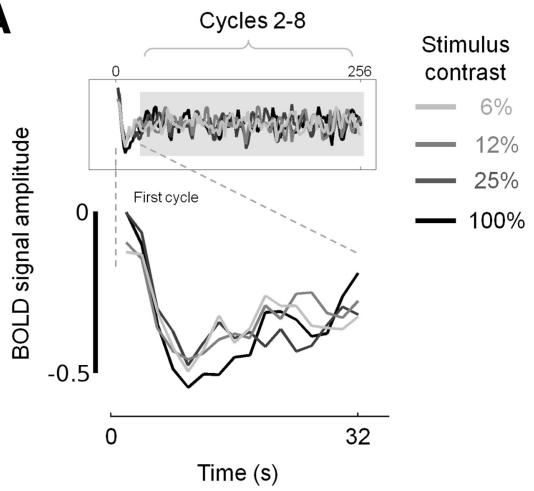

C

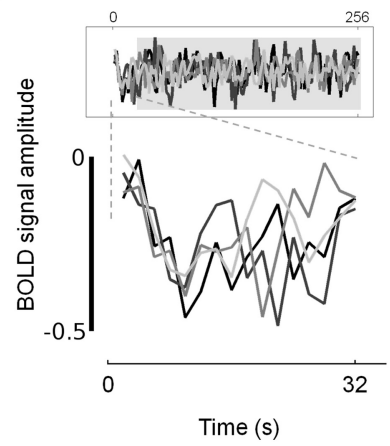

B

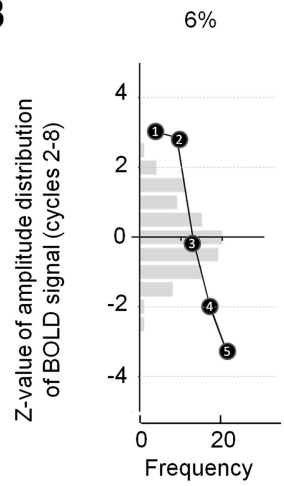

$12 \%$

$25 \%$

$100 \%$
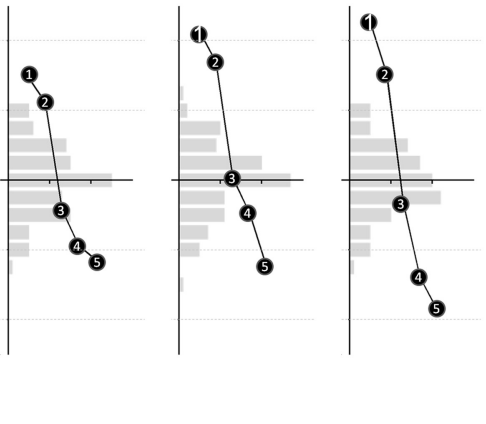

D

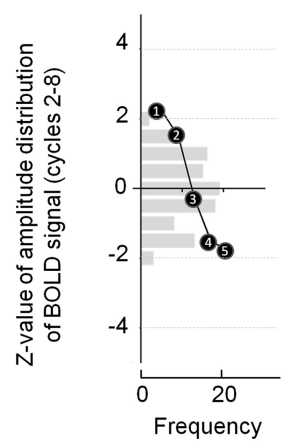

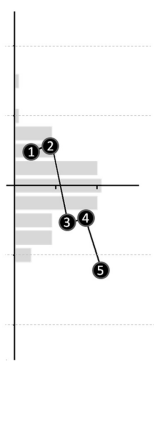

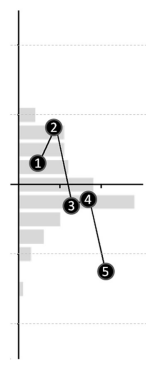

Figure 8. $\quad \boldsymbol{A}-\boldsymbol{D}$, Initial negative deflections in B0LD signals at representations of unattended locations in $\mathrm{V} 1(\boldsymbol{A}, \boldsymbol{B})$ and the $L G N(\boldsymbol{C}, \boldsymbol{D})$. $\boldsymbol{A}$, Group mean BOLD signals are plotted as a function of time for the initial stimulus cycle of $32 \mathrm{~s}$ with the full scan duration time series inset for reference. Data are plotted for the central task, which was performed throughout the scans, and for stimulus contrasts of $6,12,25$, and $100 \%$. There is a negative deflection in all time series for $\mathrm{V} 1(\boldsymbol{A})$ over the first $8-10 \mathrm{~s}$ (data samples $1-5$ at an acquisition repetition time of $2 \mathrm{~s}$ ). The signal then exhibits a modest increase, but does not recover to its initial value. The initial negative deflection is not observed in data acquired on a phantom, meaning that magnetization changes cannot explain the effects illustrated. $\boldsymbol{B}$, To assess the significance of the initial negative deflection, we compared the amplitude values of the first $10 \mathrm{~s}$ ( 5 samples) of each mean time series with the distribution of the amplitude values of the same time series after the end of the first stimulus cycle (gray shaded regions of the insets of $\boldsymbol{A}$ and $\boldsymbol{C}$, representing 33-256 s, samples 17-128). Z-transformed frequency distributions of $\mathrm{V} 1$ amplitudes for samples 17-128 are given for each stimulus contrast in light gray bars on the vertical axes. The Z-transformed amplitudes of the first five data points are overlaid on this distribution for comparison. $C, \boldsymbol{D}$, Data for the LGN were subjected to the same analysis described for V1 and are shown in the same format as $\boldsymbol{A}$ and $\boldsymbol{B}$.

Table 2. Initial negative deflection under central task conditions in LGN and V1 ipsilateral to the stimulus ${ }^{a}$

\begin{tabular}{|c|c|c|c|c|c|c|c|c|}
\hline \multirow{2}{*}{$\begin{array}{l}\text { Percentage stimulus contrast } \\
Z\end{array}$} & \multicolumn{4}{|l|}{ LGN } & \multicolumn{4}{|l|}{ V1 } \\
\hline & 6 & 12 & 25 & 100 & 6 & 12 & 25 & 100 \\
\hline Time point 1 & 2.24 & 1.01 & 1.13 & 0.65 & $2.98^{* * *}$ & $2.92^{* *}$ & $4.26^{* * *}$ & $4.53^{* * *}$ \\
\hline Time point 2 & 1.50 & 1.20 & 0.42 & $1.66^{*}$ & $2.86^{* *}$ & $2.21^{* *}$ & $3.37^{* * *}$ & $3.01^{* * *}$ \\
\hline Time point 3 & -0.31 & -1.12 & 0.33 & -0.61 & -0.13 & -0.86 & 0.07 & -0.67 \\
\hline Time point 4 & -1.54 & -0.91 & -1.22 & -0.38 & $-2.00^{*}$ & $-1.88^{*}$ & -0.94 & $-2.80^{* *}$ \\
\hline Time point 5 & $-1.74^{*}$ & $-2.40^{* *}$ & -1.40 & $-2.49^{* *}$ & $-3.28^{* * *}$ & $-2.27^{* *}$ & $-2.43^{* *}$ & $-3.68^{* * *}$ \\
\hline Gradient & $-3.15^{* * *}$ & $-2.9^{* *}$ & $-1.97^{*}$ & $-2.1^{*}$ & $-4.68^{* * *}$ & $-3.89^{* * *}$ & $-4.11^{* * *}$ & $-5.8^{* * *}$ \\
\hline
\end{tabular}

${ }^{a}$ This table summarizes the $Z$ statistic obtained by comparing BOLD amplitude at each of the five initial BOLD signal time points (samples) with the remainder of the time series (samples $17-128$ ). The bottom row of the table shows the $Z$ statistic resulting from a comparison between the gradient computed across those initial five samples with the gradient across comparable consecutive five-sample sets (taken from samples 17-128). Values with asterisks highlight significant effects between the samples $\left({ }^{*} p<0.05,{ }^{* *} p<0.01,{ }^{* * *} p<0.001\right)$.

The amplitudes obtained at temporal samples 2-4 frequently decrease sequentially between the values recorded at samples 1 and 5 . The first and fifth samples are therefore always significantly different in V1 and frequently in the LGN from samples obtained after the initial stimulus cycle (Table 2).

To characterize further the initial negative deflection in the time series, we computed the gradient over the first five samples using least-squares regression. For statistical comparison, we generated a null distribution of slopes for the rest of the dataset by iteratively sampling 108 sets of five consecutive data points from the start of the second stimulus cycle to the end of the time series. The Z-scores of the computed slopes and associated significance are given in Table 2. It is clear that in all cases the initial slope is significantly different for those computed for the remainder of the time series.

We now ask how the suppression in the representations of unattended locations in the LGN might arise. One potential source of suppression in the LGN is feedback from V1. However, the LGN also has connections to other subcortical structures that could play a role. Specifically, the pulvinar and superior colliculus, which have been implicated in attention and connect directly or indirectly with the LGN (Saalmann and Kastner, 2009), may also contribute to suppression in the LGN. To investigate the role of these structures, we performed a prospective whole-brain analysis on data acquired in our initial experiments and in the $100 \%$ contrast condition of the follow-up experiments. The pos- 

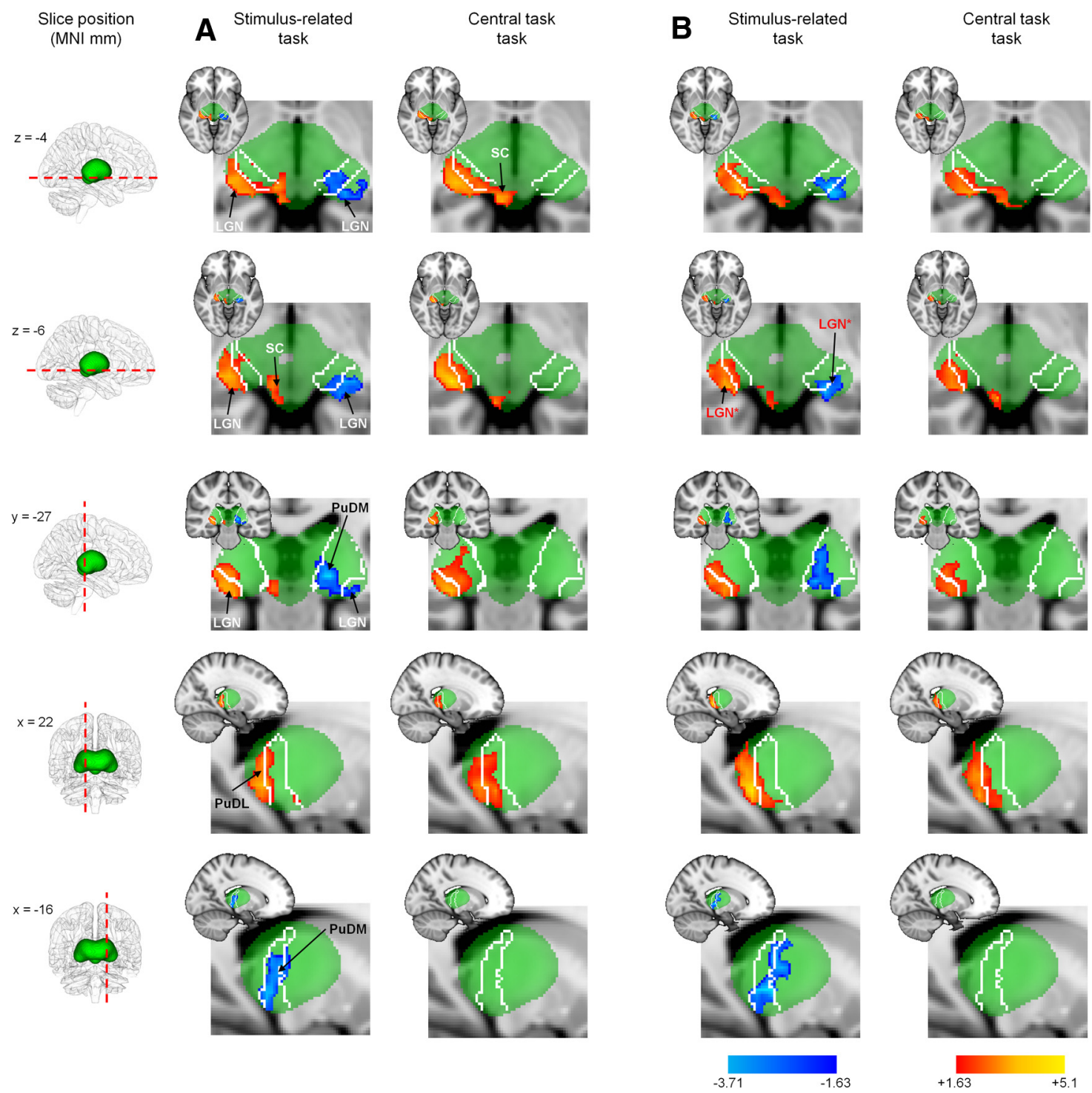

Figure 9. Patterns of thalamic activation and suppression under different task conditions. $A, B, Z$-score maps for stimulus $>$ no stimulus (hot colors) and stimulus $<$ no stimulus (cool colors) for our initial experiments $(\boldsymbol{A})$ and for the $100 \%$ contrast condition in our follow-up experiment $(\boldsymbol{B})$. Data are shown for axial (top 2 rows), coronal (middle row), and sagittal (bottom 2 rows) sections. These results were thresholded using clusters determined by $Z>1.63$ and a cluster-corrected significance threshold of $p<0.05$; note also that no small-volume correction has been applied. The green region depicts the extent of a standard thalamic probability atlas described in MNI space; the white outline drawn within the thalamus depicts the extent of the region with the greatest connectivity probability to parietal regions (see Materials and Methods). The data in $\boldsymbol{A}$ and $\boldsymbol{B}$ exhibit remarkably similar features: activation is restricted to the LGN, SC, and dorsolateral pulvinar (PuDL) contralateral to the stimulus under both task conditions, while suppression is observed in the both LGN and dorsomedial pulvinar (PuDM) ipsilateral to the stimulus but only under stimulus-related task conditions. In $\boldsymbol{B}$, the region labeled LGN* (in red) highlights the position of the centers of the LGN ROls identified in a previous study (Kastner et al., 2004).

itive and negative signals in subcortical structures for the stimulus-related and central tasks are shown in Figure 9. The first striking feature of the analysis is that the results obtained in the follow-up experiment (Fig. 9B) almost perfectly replicate those of the initial experiments (Fig. 9A). The details of the results are discussed with respect to the initial experiment only. First, consistent with our ROI analysis, the positive and negative signals in the LGN are evident contralateral and ipsilateral to the stimulus, respectively. As expected the negative signals are only detected during the stimulus-related task. Importantly, the LGN regions highlighted by the analysis coincide with our anatomically defined LGN ROIs and previously published coordinates (Fig. 9B) for the LGN (Kastner et al., 2004). Second, the superior colliculus and the dorsolateral aspects of the pulvinar contralateral to the stimulus exhibit significant positive responses under both task conditions. Third, the dorsomedial aspects of the pulvinar ipsilateral to the stimulus exhibit negative responses during the stimulus-related task. It is important to note that the pulvinar responses found contralateral and ipsilateral to the stimulus occupy distinct and separate aspects of the pulvinar, which have connections to different cortical locations. The dorsolateral pulvinar has a greater proportion of occipital projections than the dorsomedial pulvinar, which connects preferentially to parietal cortex (Gutierrez et al., 2000; Behrens et al., 2003).

Given the consistency between the patterns of responses obtained for the two separate experiments (Fig. 9, compare $A, B$ ), we used the significant clusters of positive and negative responses obtained from the group analysis of the initial experiment (Fig. $9 A$ ) as independent ROIs for extracting contrast responses for the superior colliculus, dorsolateral pulvinar contralateral to the stimulus, and the dorsomedial pulvinar ipsilateral to the stimu- 
A Dorsomedial pulvinar (ipsilateral)
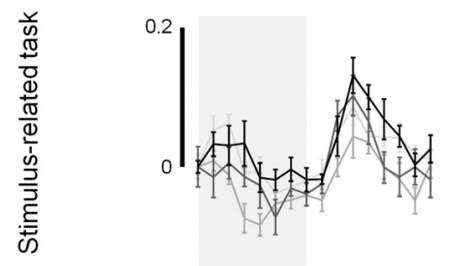

B Dorsolateral pulvinar (contralateral)
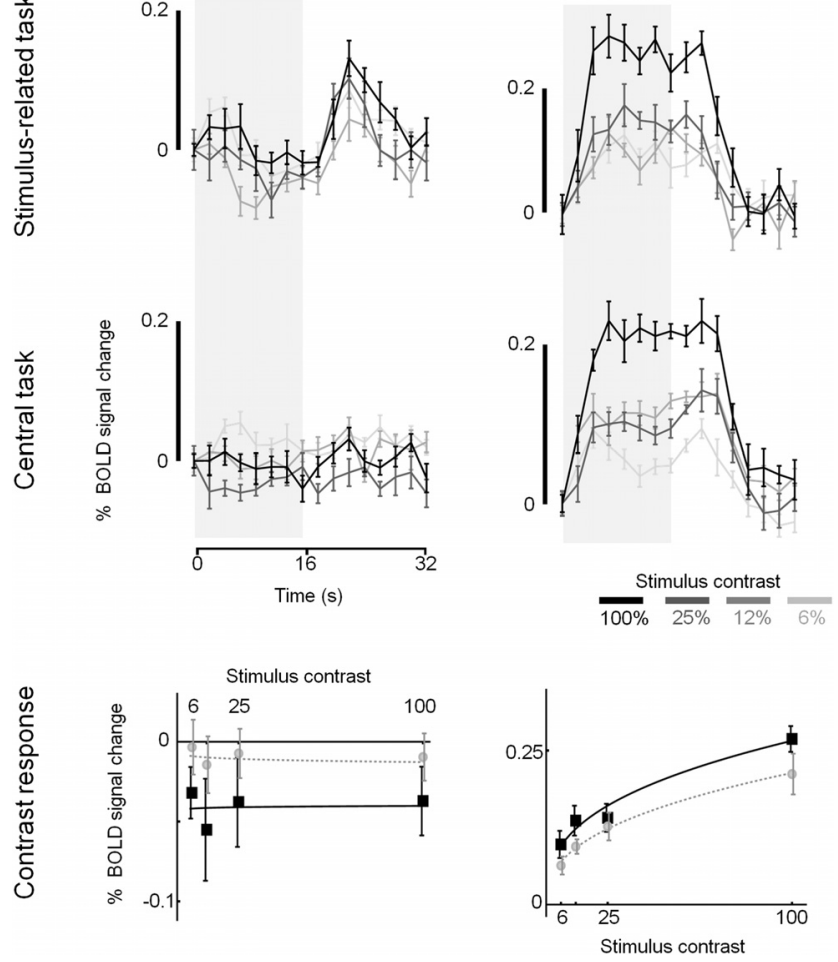

Figure 10. Group mean BOLD time series (top 2 rows) and contrast response functions (bottom row). $A, B$, Data are given for $(\boldsymbol{A})$ the dorsomedial pulvinar ipsilateral to the stimulus and $(\boldsymbol{B})$ the dorsolateral pulvinar contralateral to the stimulus. Time series data are given for four contrast levels for stimulus-related task (top row) and central task (middle row), and were generated and displayed as described in Figure 3. Scale bar to the left of each plot indicates $0.2 \%$ BOLD signal change. Contrast response functions (bottom row) are shown for the stimulusrelated task (black squares) and central tasks (gray circles).

lus. The ROIs were back-projected into the individual subject anatomies using the inverse affine transformations calculated in the alignment of the functional data to the individual subject anatomy, and subsequently to the MNI template brain. Following back-projection of the ROIs into the individual subject coordinate space, the contiguous clusters reaching statistical significance for both the dorsolateral and dorsomedial pulvinar ROIs fell within the anatomical bounds of the thalamus, and thus were not subjected to further restriction. Back-projection of the superior colliculus ROIs sometimes resulted in individual subject ROIs capturing voxels representing CSF rather than brain tissue. Functional MRI measurements from the superior colliculus are known to be particularly susceptible to physiological noise sources (Wall et al., 2009) partly due to the fact that the structure lies immediately adjacent to the CSF. Taking direction from previous studies (Limbrick-Oldfield et al., 2012), we took the precaution of restricting the back-projected superior colliculus ROIs to brain matter, manually excluding any voxels corresponding to CSF. This resulted in superior colliculus ROIs of a mean volume of $41.2 \pm 7.1 \mathrm{~mm}^{3}$, which is consistent with that previously reported by Schneider and Kastner (2009). The mean center of mass of the revised superior colliculus ROIs was at MNI coordinates $x=6, y=-32, z=-4$, which lies in the center of a superior colliculus ROI previously reported (Limbrick-Oldfield et al., 2012).

The time series and contrast response functions for the dorsomedial and dorsolateral pulvinar ROIs, where responses were most significant, are shown in Figure 10. There appears to be an effect of task, but not contrast, in the dorsomedial pulvinar, and contrast, but not task, in the dorsolateral pulvinar. As for the LGN, V1, and V5 data previously described, we use repeatedmeasures ANOVA approaches with task and stimulus contrast as main effects to assess these data from the pulvinar along with those derived from the superior colliculus ROIs. In the dorsomedial pulvinar ipsilateral to the stimulus, a main effect of task $\left(F_{(1,7)}\right.$ $=8.009, p=0.025)$, but not contrast $\left(F_{(3,21)}=0.624, p=0.607\right)$, was evident (Fig. 10A). There was no interaction between contrast and task $\left(F_{(3,21)}=0.085, p=0.967\right)$. This is the pattern of response we found in the LGN and V1 ipsilateral to the stimulus. The dorsolateral pulvinar contralateral to the stimulus (Fig. 10B) exhibited responses that were dependent on contrast $\left(F_{(3,21)}=\right.$ $\left.25.08, p=3.872 \times 10^{-7}\right)$ but not task $\left(F_{(1,7)}=2.800, p=0.138\right)$ and there was no interaction $\left(F_{(3,21)}=0.633, p=0.602\right)$. The results are therefore consistent with those we measured in the LGN contralateral to the stimulus. In the superior colliculus contralateral to the stimulus, the responses were influenced by task $\left(F_{(1,7)}=6.318, p=0.040\right)$ but not contrast $\left(F_{(3,21)}=1.575, p=\right.$ $0.23)$. No interaction was evident $\left(F_{(3,21)}=0.521, p=0.673\right)$.

A clear feature of the positive BOLD responses we obtained from the stimulus representations in the LGN, V1, and V5 is an increasing task dependence, but reducing contrast dependence as we move up the visual hierarchy, a pattern of results consistently reported in single-unit, evoked-potential, and fMRI studies of attention (for review, see Carrasco, 2011). A recent Normalization Model of Attention (Reynolds and Heeger, 2009; Boynton, 2011) successfully predicts qualitatively different effects of attention on cortical responses that have been reported in the literature. The key feature of this model is an early "attention field" that acts before cortical responses are normalized. The model also has the following advantages: (1) the effect of multiple stimuli of different sizes and locations can be accounted for; (2) the model allows manipulation of the spatial parameters of an attention field as well as receptive fields, allowing the modeling of effects in different visual areas; (3) the receptive fields are modeled by taking the extent of both the excitatory center and suppressive surround into account.

Reynolds and Heeger (2009) showed that when the attention field is large compared with the visual stimulus, a contrast gain effect of attention is observed, but when the attention field is comparable in size to the stimulus, the result is a response gain attentional effect (see Table 3). Importantly, the authors kept the receptive field properties constant because they were modeling the effect of attention in a single visual area. Here, we determined whether the model can account for the response characteristics we observed in two different visual areas-V1 and V5. To account for attentional effects in V1 and V5, we varied the receptive field parameters of the model, while necessarily holding the attention field and stimulus parameters fixed (Table 3). For V1, therefore, we used relatively small receptive fields compared with V5. We also modified the attention field, which in our case now suppresses unattended locations rather than facilitating attended locations as used by default in the model. As can be seen in Figure 11 , this approach yields contrast responses that adequately capture the type of responses we measured. For V1, where receptive fields are small relative to the attention field, attention to the large lateralized stimulus produces a leftward shift in the contrast response function, analogous to a contrast gain-type mechanism. For V5, where receptive fields are comparable in size to the attention field, attention produces a response modulation that is carried largely by a response gain-type mechanism. Accordingly, a single, early attention field that acts by suppressing unattended 
Table 3. Model parameters ${ }^{a}$

\begin{tabular}{llllll}
\hline Model description & Stimulus 1 size & Stimulus 2 size & Attention field size & Excitatory center size & Inhibitory surround size \\
\hline Reynolds and Heeger, Fig. 2A & 3 & 3 & 30 & 5 & 20 \\
Reynolds and Heeger, Fig 2B & 5 & 5 & 3 & 5 & 20 \\
V1 & $50.4^{b}$ & $1.3^{b}$ & 30 & 1 & 4 \\
V5 & $50.4^{b}$ & $1.3^{b}$ & 30 & 15 & 60 \\
\hline
\end{tabular}

${ }^{a}$ Summary of the input parameters (arbitrary units, only relative sizes are meaningful) to "The Normalization Model of Attention" (Reynolds and Heeger, 2009). For context, the top two rows summarize the values used by Reynolds and Heeger to introduce the model and its effects (Reynolds and Heeger, 2009; their Fig. 2A, B). The bottom two rows show the model input parameters representing our stimuli and the receptive field sizes of V1 and V5. We used sigma values (see Reynolds and Heeger, 2009, for description) of $7.5 \times 10^{-5}$ for V1 and $10^{-5}$ for V5 to account for differences in the well documented compressiveness between the contrast response functions of V1 and V5.

${ }^{b}$ Scaled by 3.6 units per degree of visual angle; see text for explanation.

\section{A}

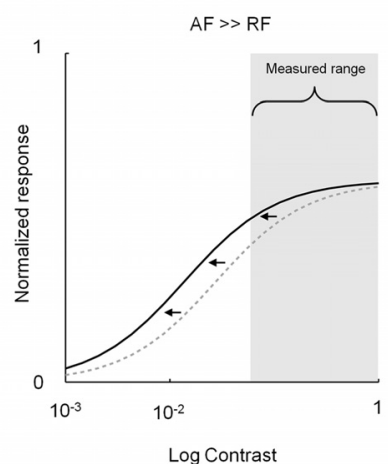

Log Contrast
B

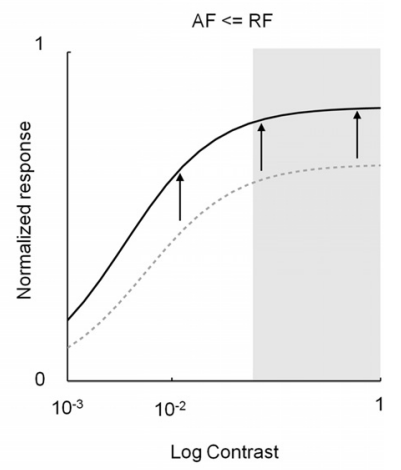

........... Unattended - Attended

Figure 11. The Normalization Model of Attention: predicted effects in V1 and V5. $\boldsymbol{A}, \boldsymbol{B}$, Normalized model-predicted contrast responses (based on the input parameters defined in Table 3) are plotted for V1 (A) and V5 (B). The relatively large attention field (AF) to receptive field (RF) size ratio in V1 $(\boldsymbol{A})$ results in contrast gain at the attended locations. For V5 $(\boldsymbol{B})$, the lower AF to RF size ratio results in an effect of attention that is carried by a response gain-type mechanism. Note that these effects result from modeling the AF as suppressive at unattended locations rather than enhancing responses at attended locations. Note also that the portion of the model-predicted contrast response overlapping our sampled empirical data range (contrast, $6-100 \%$ ) is qualitatively very similar to our measured data for V1 (compare Fig. $11 \mathrm{~A}$ with Fig. $7 B$ ) and V5 (compare Fig. 11B with Fig. 7C).

locations can account for the types of response we observe in conjunction with known differences in receptive field properties of neurons in V1 and V5. The data presented in Figures 9 and 10 suggest that a suppressive, early attention field may reside in the pulvinar.

\section{Discussion}

Our new, inter-related findings shed light on how the human brain represents visual information under different task and stimulus conditions. The key finding we make is that in the representations of unattended locations, negative BOLD signals are present and show little evidence of stimulus-contrast dependence. These negative signals are found in a network of brain areas, including both cortical (V1) and subcortical (LGN and pulvinar) structures. Moreover, we were able to disambiguate negative BOLD signals from signals arising from blinks, which are seldom considered as a potential confound (but see Hupé et al., 2012). Given the evidence that links negative BOLD responses to other physiological markers of neural suppression (Goense et al., 2012; Mullinger et al., 2014), we discuss our results in terms of the suppressive effects of attention. An autoradiography study in macaque (Vanduffel et al., 2000) showed suppression of the both the LGN and V1 at representations of unattended locations, features we reproduced in human and characterized further. Previous human fMRI studies showed suppression in V1 (Tootell et al., 1998a; Smith et al., 2000; Shmuel et al., 2002; Wade and
Rowland, 2010) and some, most relevant to our study, explored suppression specifically in the hemisphere ipsilateral to a lateralized visual stimulus (Tootell et al., 1998a; Smith et al., 2000). However, even these most-relevant fMRI studies examined responses under a single stimulus and task condition. Moreover, the study on macaque first showing suppression in the LGN and V1 effectively used one stimulus condition with two tasks. We set out therefore to characterize suppression as a function of task and stimulus contrast in the LGN and V1 and found that suppression is highly task-dependent, but depends very little on stimulus contrast. The suppression we observed is also consistent with reported attention-related changes of baseline firing of neurons that are not stimulated: the firing rates of V4 and V2, but not V1, neurons with receptive fields that do not represent the stimulus decrease when attention is directed outside compared with inside the receptive field (Luck et al., 1997). Overall, therefore, our results highlight a task-dependent suppression of neural activity in very early visual structures as an important component of attention.

Our study also characterized how task affects positive signals as a function of contrast at stimulus representations in the LGN and V1. A key finding is that the stimulus-driven activity within the stimulus representation in the LGN does not increase when stimuli are attended. This is consistent with single-unit (Mehta et al., 2000a,b) and autoradiography measurements on macaque (Vanduffel et al., 2000). An early fMRI study demonstrated large attention-related increases in the activity of the human LGN (O'Connor et al., 2002), but more recent studies have found more modest attention-related changes (Schneider and Kastner, 2009; Schneider, 2011). Our results seem more consistent with the recent work and can be largely reconciled with the earlier study on the basis of methodological differences. By alternating stimulation from one hemifield to the other, without a baseline condition, the approach used by O'Connor et al. (2002) could capture both modest attention-dependent increases (Schneider and Kastner, 2009), along with perhaps larger effects reflecting a release from suppression that we measure here. We did, however, detect an attention-dependent increase in the superior colliculus contralateral to the stimulus, consistent with literature highlighting an attentional role of this structure (Schneider and Kastner, 2009; Katyal et al., 2010; Lovejoy and Krauzlis, 2010; Schneider, 2011; Krauzlis et al., 2013; Katyal and Ress, 2014).

The stimulus-driven positive signals in V1 that we measured were larger when the stimulus is attended, consistent with the fMRI literature. This effect is most obvious at intermediate and low contrasts, a finding replicated across multiple studies (Martínez et al., 1999; Somers et al., 1999; Buracas and Boynton, 2007; Li et al., 2008). At high contrast, we find no attention-related increase in responses at the stimulus representation, consistent with one study (Li et al., 2008), but not another (Gandhi et al., 1999). It is possible that if we increased our task difficulty (our tasks were easier than those used in other studies), an effect of 
attention on V1 signals and indeed the LGN might emerge across all contrasts. In any case, it is clear that both baseline shifts (Ress et al., 2000; Murray, 2008) and contrast gain appear to interact in early visual areas under different task conditions to give rise to differing overall effects of attention on fMRI contrast response functions (Li et al., 2008).

The attention-dependent suppression we reveal in the LGN is unlikely to originate there. We found two other structures, V1 and the dorsomedial pulvinar, that also exhibit suppression. V1 has extensive feedback connections to LGN and the pulvinar, so it could be the source of suppression in both thalamic structures. However, it is also plausible that the pulvinar is the source of suppression in V1 and the LGN and there is direct evidence for the former (Purushothaman et al., 2012). The pulvinar has long been associated with attention (Chalupa et al., 1976). Also, human lesion (Rafal and Posner, 1987; Snow et al., 2009) and neuroimaging studies (Kastner et al., 2004; Cotton and Smith, 2007) have documented the involvement of the pulvinar in attention. Particularly relevant to our study, attentional deficits have also been reported in the hemifield contralateral to the hemisphere in which the pulvinar was inhibited (Desimone et al., 1990). The pulvinar also has feedforward and feedback connections with occipital, parietal, and frontal cortex (Shipp, 2003). The reductions in signals we observe during the stimulus-related task are largely restricted to the aspects of the pulvinar that connect strongly, but not exclusively, to the parietal cortex (Gutierrez et al., 2000; Behrens et al., 2003). It is possible therefore that the attention-related suppression arises in parietal cortex and is fed back to the pulvinar, which in turn influences signaling in the LGN and V1. The conduit for influencing signals in the LGN may be via V1 or perhaps the thalamic reticular nucleus, which could act as the intermediary that links the suppression we observe in the pulvinar to the LGN (McAlonan et al., 2008; Saalmann and Kastner, 2009). Our responses within the stimulus representations in the pulvinar do not appear to be modulated by attention. It is also clear that these stimulus representations do not occupy the same regions of the pulvinar as those that are suppressed by attention in the opposite hemisphere. It is important therefore to consider the subdivisions of the pulvinar because they appear to play different roles with respect to stimulus and task conditions (Robinson and Petersen, 1992; Shipp, 2003).

Given the suppression we find in subcortical areas, we asked whether a leading model of attention that invokes an early attention field can account for our results. The Normalization Model of Attention (Reynolds and Heeger, 2009), with the modification of a suppressive rather than facilitatory attention field, was able to account for the types of response we observed in V1 and V5. While we have shown that early suppression in an attention field can adequately account for our responses in V1 and V5, it may also play a role in other attentional effects. For example, others have put forward the idea that shifts in V5 receptive field location can be explained on the basis of the attentional suppression of some but not all of V1's inputs to V5 neurons (Anton-Erxleben et al., 2009), for which we provide evidence here.

We have shown that suppression is found in the representations of unattended locations of the LGN and V1. Similar effects were also found in the pulvinar. Importantly, these attentionrelated decreases in neural response carried little or no information about the attended stimulus. It is clear therefore that suppression, most notably in subcortical structures, plays an important role in spatial attention in human. Our findings have a number of important implications. First, suppression is an expression of negative feedback, a system that is both more stable and energetically efficient than excitatory, positive feedback systems (Niven and Laughlin, 2008). Second, a leading model of attention (Reynolds and Heeger, 2009) proposes the concept of a low-level attention field, which our results indicate could be suppressive and could reside in the pulvinar. Related to both points is the fact that large increases in neural responses in extrastriate visual cortex can result from relatively small, but widespread suppressive effects in antecedent visual structures.

\section{References}

Allman JM, Kaas JH (1971) A representation of the visual field in the caudal third of the middle tempral gyrus of the owl monkey (Aotus trivirgatus). Brain Res 31:85-105. CrossRef Medline

Amano K, Wandell BA, Dumoulin SO (2009) Visual field maps, population receptive field sizes, and visual field coverage in the human $\mathrm{MT}+\mathrm{com}-$ plex. J Neurophysiol 102:2704-2718. CrossRef Medline

Andrews TJ, Halpern SD, Purves D (1997) Correlated size variations in human visual cortex, lateral geniculate nucleus, and optic tract. J Neurosci 17:2859-2868. Medline

Anton-Erxleben K, Stephan VM, Treue S (2009) Attention reshapes centersurround receptive field structure in macaque cortical area MT. Cereb Cortex 19:2466-2478. CrossRef Medline

Baseler HA, Gouws A, Haak KV, Racey C, Crossland MD, Tufail A, Rubin GS, Cornelissen FW, Morland AB (2011) Large-scale remapping of visual cortex is absent in adult humans with macular degeneration. Nat Neurosci 14:649-655. CrossRef Medline

Behrens TE, Johansen-Berg H, Woolrich MW, Smith SM, Wheeler-Kingshott CA, Boulby PA, Barker GJ, Sillery EL, Sheehan K, Ciccarelli O, Thompson AJ, Brady JM, Matthews PM (2003) Non-invasive mapping of connections between human thalamus and cortex using diffusion imaging. Nat Neurosci 6:750-757. CrossRef Medline

Boynton GM (2011) Spikes, BOLD, attention, and awareness: a comparison of electrophysiological and fMRI signals in V1. J Vis 11(5):12. CrossRef Medline

Buracas GT, Boynton GM (2007) The effect of spatial attention on contrast response functions in human visual cortex. J Neurosci 27:93-97. CrossRef Medline

Carrasco M (2011) Visual attention: the past 25 years. Vision Res 51:14841525. CrossRef Medline

Chalupa LM, Coyle RS, Lindsley DB (1976) Effect of pulvinar lesions on visual pattern discrimination in monkeys. J Neurophysiol 39:354-369. Medline

Cotton PL, Smith AT (2007) Contralateral visual hemifield representations in the human pulvinar nucleus. J Neurophysiol 98:1600-1609. CrossRef Medline

Desimone R, Wessinger M, Thomas L, Schneider W (1990) Attentional control of visual perception: cortical and subcortical mechanisms. Cold Spring Harb Symp Quant Biol 55:963-971. CrossRef Medline

Devlin JT, Sillery EL, Hall DA, Hobden P, Behrens TE, Nunes RG, Clare S, Matthews PM, Moore DR, Johansen-Berg H (2006) Reliable identification of the auditory thalamus using multi-modal structural analyses. Neuroimage 30:1112-1120. CrossRef Medline

Dumoulin SO, Bittar RG, Kabani NJ, Baker CL Jr, Le Goualher G, Bruce Pike G, Evans AC (2000) A new anatomical landmark for reliable identification of human area V5/MT: a quantitative analysis of sulcal patterning. Cereb Cortex 10:454-463. CrossRef Medline

Duncan J (2006) EPS Mid-Career Award 2004: brain mechanisms of attention. Q J Exp Psychol (Hove) 59:2-27. CrossRef Medline

Fujita N, Tanaka H, Takanashi M, Hirabuki N, Abe K, Yoshimura H, Nakamura H (2001) Lateral geniculate nucleus: anatomic and functional identification by use of MR imaging. AJNR Am J Neuroradiol 22:17191726. Medline

Gandhi SP, Heeger DJ, Boynton GM (1999) Spatial attention affects brain activity in human primary visual cortex. Proc Natl Acad Sci U S A 96: 3314-3319. CrossRef Medline

Goense J, Merkle H, Logothetis NK (2012) High-resolution fMRI reveals laminar differences in neurovascular coupling between positive and negative BOLD responses. Neuron 76:629-639. CrossRef Medline

Gouws A, Woods W, Millman R, Morland A, Green G (2009) DataViewer3D: an open-source, cross-platform multi-modal neuroimaging data visualization tool. Front Neuroinform 3:9. CrossRef Medline 
Gutierrez C, Cola MG, Seltzer B, Cusick C (2000) Neurochemical and connectional organization of the dorsal pulvinar complex in monkeys. J Comp Neurol 419:61-86. CrossRef Medline

Hupé JM, Bordier C, Dojat M (2012) A BOLD signature of eyeblinks in the visual cortex. Neuroimage 61:149-161. CrossRef Medline

Jenkinson M, Bannister P, Brady M, Smith S (2002) Improved optimization for the robust and accurate linear registration and motion correction of brain images. Neuroimage 17:825-841. CrossRef Medline

Kastner S, Pinsk MA, De Weerd P, Desimone R, Ungerleider LG (1999) Increased activity in human visual cortex during directed attention in the absence of visual stimulation. Neuron 22:751-761. CrossRef Medline

Kastner S, O'Connor DH, Fukui MM, Fehd HM, Herwig U, Pinsk MA (2004) Functional imaging of the human lateral geniculate nucleus and pulvinar. J Neurophysiol 91:438-448. Medline

Katyal S, Ress D (2014) Endogenous attention signals evoked by threshold contrast detection in human superior colliculus. J Neurosci 34:892-900. CrossRef Medline

Katyal S, Zughni S, Greene C, Ress D (2010) Topography of covert visual attention in human superior colliculus. J Neurophysiol 104:3074-3083. CrossRef Medline

Krauzlis RJ, Lovejoy LP, Zénon A (2013) Superior colliculus and visual spatial attention. Annu Rev Neurosci 36:165-182. CrossRef Medline

Li X, Lu ZL, Tjan BS, Dosher BA, Chu W (2008) Blood oxygenation leveldependent contrast response functions identify mechanisms of covert attention in early visual areas. Proc Natl Acad Sci U S A 105:6202-6207. CrossRef Medline

Limbrick-Oldfield EH, Brooks JC, Wise RJ, Padormo F, Hajnal JV, Beckmann CF, Ungless MA (2012) Identification and characterisation of midbrain nuclei using optimised functional magnetic resonance imaging. Neuroimage 59:1230-1238. CrossRef Medline

Lovejoy LP, Krauzlis RJ (2010) Inactivation of primate superior colliculus impairs covert selection of signals for perceptual judgments. Nat Neurosci 13:261-266. CrossRef Medline

Luck SJ, Chelazzi L, Hillyard SA, Desimone R (1997) Neural mechanisms of spatial selective attention in areas V1, V2, and V4 of macaque visual cortex. J Neurophysiol 77:24-42. Medline

Martínez A, Anllo-Vento L, Sereno MI, Frank LR, Buxton RB, Dubowitz DJ, Wong EC, Hinrichs H, Heinze HJ, Hillyard SA (1999) Involvement of striate and extrastriate visual cortical areas in spatial attention. Nat Neurosci 2:364-369. CrossRef Medline

McAlonan K, Cavanaugh J, Wurtz RH (2008) Guarding the gateway to cortex with attention in visual thalamus. Nature 456:391-394. CrossRef Medline

Mehta AD, Ulbert I, Schroeder CE (2000a) Intermodal selective attention in monkeys. II: physiological mechanisms of modulation. Cereb Cortex 10: 359-370. CrossRef Medline

Mehta AD, Ulbert I, Schroeder CE (2000b) Intermodal selective attention in monkeys. I: distribution and timing of effects across visual areas. Cereb Cortex 10:343-358. CrossRef Medline

Motter BC (1993) Focal attention produces spatially selective processing in visual cortical areas $\mathrm{V} 1, \mathrm{~V} 2$, and $\mathrm{V} 4$ in the presence of competing stimuli. J Neurophysiol 70:909-919. Medline

Mullinger KJ, Mayhew SD, Bagshaw AP, Bowtell R, Francis ST (2014) Evidence that the negative BOLD response is neuronal in origin: a simultaneous EEG-BOLD-CBF study in humans. Neuroimage 94:263-274. CrossRef Medline

Murray SO (2008) The effects of spatial attention in early human visual cortex are stimulus independent. J Vis 8(10):2.1-11. CrossRef Medline

Niven JE, Laughlin SB (2008) Energy limitation as a selective pressure on the evolution of sensory systems. J Exp Biol 211:1792-1804. CrossRef Medline

O'Connor DH, Fukui MM, Pinsk MA, Kastner S (2002) Attention modulates responses in the human lateral geniculate nucleus. Nat Neurosci 5:1203-1209. CrossRef Medline
Posner MI (1980) Orienting of attention. Q J Exp Psychol 32:3-25. CrossRef Medline

Purushothaman G, Marion R, Li K, Casagrande VA (2012) Gating and control of primary visual cortex by pulvinar. Nat Neurosci 15:905-912. CrossRef Medline

Rafal RD, Posner MI (1987) Deficits in human visual spatial attention following thalamic lesions. Proc Natl Acad Sci U S A 84:7349-7353. CrossRef Medline

Ress D, Backus BT, Heeger DJ (2000) Activity in primary visual cortex predicts performance in a visual detection task. Nat Neurosci 3:940-945. CrossRef Medline

Reynolds JH, Heeger DJ (2009) The normalization model of attention. Neuron 61:168-185. CrossRef Medline

Robinson DL, Petersen SE (1992) The pulvinar and visual salience. Trends Neurosci 15:127-132. CrossRef Medline

Saalmann YB, Kastner S (2009) Gain control in the visual thalamus during perception and cognition. Curr Opin Neurobiol 19:408-414. CrossRef Medline

Schneider KA (2011) Subcortical mechanisms of feature-based attention. J Neurosci 31:8643-8653. CrossRef Medline

Schneider KA, Kastner S (2009) Effects of sustained spatial attention in the human lateral geniculate nucleus and superior colliculus. J Neurosci 29: 1784-1795. CrossRef Medline

Shipp S (2003) The functional logic of cortico-pulvinar connections. Philos Trans R Soc Lond B Biol Sci 358:1605-1624. CrossRef Medline

Shmuel A, Yacoub E, Pfeuffer J, Van de Moortele PF, Adriany G, Hu X, Ugurbil K (2002) Sustained negative BOLD, blood flow and oxygen consumption response and its coupling to the positive response in the human brain. Neuron 36:1195-1210. CrossRef Medline

Slotnick SD, Schwarzbach J, Yantis S (2003) Attentional inhibition of visual processing in human striate and extrastriate cortex. Neuroimage 19: 1602-1611. CrossRef Medline

Smith AT, Singh KD, Greenlee MW (2000) Attentional suppression of activity in the human visual cortex. Neuroreport 11:271-277. CrossRef Medline

Smith AT, Williams AL, Singh KD (2004) Negative BOLD in the visual cortex: evidence against blood stealing. Hum Brain Mapp 21:213-220. CrossRef Medline

Snow JC, Allen HA, Rafal RD, Humphreys GW (2009) Impaired attentional selection following lesions to human pulvinar: evidence for homology between human and monkey. Proc Natl Acad Sci U S A 106:4054-4059. CrossRef Medline

Somers DC, Dale AM, Seiffert AE, Tootell RB (1999) Functional MRI reveals spatially specific attentional modulation in human primary visual cortex. Proc Natl Acad Sci U S A 96:1663-1668. CrossRef Medline

Tootell RB, Mendola JD, Hadjikhani NK, Liu AK, Dale AM (1998a) The representation of the ipsilateral visual field in human cerebral cortex. Proc Natl Acad Sci U S A 95:818-824. CrossRef Medline

Tootell RB, Hadjikhani N, Hall EK, Marrett S, Vanduffel W, Vaughan JT, Dale AM (1998b) The retinotopy of visual spatial attention. Neuron 21: 1409-1422. CrossRef Medline

Treue S, Martinez-Trujillo JC (2007) Attending to features inside and outside the spotlight of attention. Neuron 55:174-176. CrossRef Medline

Vanduffel W, Tootell RB, Orban GA (2000) Attention-dependent suppression of metabolic activity in the early stages of the macaque visual system. Cereb Cortex 10:109-126. CrossRef Medline

Wade AR, Rowland J (2010) Early suppressive mechanisms and the negative blood oxygenation level-dependent response in human visual cortex. J Neurosci 30:5008 -5019. CrossRef Medline

Wall MB, Walker R, Smith AT (2009) Functional imaging of the human superior colliculus: an optimised approach. Neuroimage 47:1620-1627. CrossRef Medline

Zeki SM (1969) The secondary visual areas of the monkey. Brain Res 13: 197-226. CrossRef Medline 